\title{
Champagne, beer, or coffee? A corpus of gender- related and neutral words
}

\author{
JARRET T. CRAWFORD \\ Rutgers University, Piscataway, New Jersey \\ P. ANDREW LEYNES \\ College of New Jersey, Ewing, New Jersey \\ CHRISTOPHER B. MAYHORN \\ North Carolina State University, Raleigh, North Carolina \\ and \\ MARTIN L. BINK \\ Western Kentucky University, Bowling Green, Kentucky
}

\begin{abstract}
A list of gender-related and gender-neutral words for use in testing gender stereotyping and memory was created and evaluated. Words were rated by samples of undergraduates at universities located in the northeast, southeast, and south-central United States. A substantial list of masculine, feminine, and gender-neutral words was identified. These lists allow researchers to construct large lists of genderassociated words while being able to control for extraneous variables, such as word frequency and word length. In addition, the high reliability across the samples suggests that gender ratings are a fairly stable phenomenon. Applications for this list are discussed. The word lists presented in Tables 1-3 and the raw data analyzed in this article may be downloaded from www.psychonomic.org/archive/.
\end{abstract}

The study of the nature of stereotypes has a long history in the social sciences (Allport, 1954; Katz \& Braly, 1933). The social cognitive perspective (Schneider, 1996), which focuses on the cognitive processes that underlie the categorization of individuals with regard to race, gender, sexual orientation, political affiliation, attractiveness, and other determining factors (cf. Bessenoff \& Sherman, 2000; Greenwald \& Banaji, 1995; Macrae, Mitchell, \&, Pendry, 2002; Mather, Johnson, \& De Leonardis, 1999) has provided the dominant paradigm for studying stereotypes. In the studies listed above and in others, the relationship between category-specific characteristics and/or behaviors (i.e., stereotypes) and aspects of human memory has been investigated. Many researchers have explored memory and gender stereotypes in particular. Banaji and Greenwald (1995) examined the misattribution of fame to familiar and nonfamiliar forenames. Participants were exposed to an equal number of male and female forenames attached to common last names (e.g., Peter Walker or Susan Walker). When tested at a later time, the participants were more likely to attribute fame to masculine names. These results show evidence of a bias to associate male gender, rather than female gender, to achievement or fame. Similarly,

Correspondence concerning this article should be addressed to J.T. Crawford, Department of Psychology, Rutgers University, 53 Avenue E, Piscataway, NJ 08854 (e-mail: jarretc@eden.rutgers.edu).
Macrae, Alnwick, Milne, and Schloerscheidt (2002) have presented evidence that women use more male stereotype information in semantic memory during particular phases of the menstrual cycle.

Despite these intriguing results, one of the challenges facing researchers in gender stereotypes and memory is developing an extensive list of gender-stereotypical items that can be used for additional investigations. Although some investigators have compiled lists of gender-specific items and behaviors (Bem, 1974; Blair \& Banaji, 1996; Carreiras, Garnham, Oakhill, \& Cain, 1996; Edelbrock \& Sugawara, 1978), these lists lack the scope necessary for the research methodology of memory testing. Between these studies, fewer than 50 words have been identified as stereotypically male, female, or gender neutral. Although Rosenkrantz, Vogel, Bee, Broverman, and Broverman (1968) compiled a 122-item list of differentiated male and female words and traits, these items are not necessarily representative, because the criterion for selection of male and female items was agreement between just 2 participants. In addition, their list included phrases (e.g., kind to animals), which would be difficult to adapt to many memory investigations in a way that would control for extraneous variables that are known to affect remembering, such as word frequency (e.g., Deese, 1961), word length (e.g., Baddeley, Thomson, \& Buchanan, 1975), or concreteness (e.g., Paivio, 1969). One of the more promising endeavors in the investigation of cognition has been the relatively recent appli- 
cation of brain-imaging technology. All of the current brain-imagining methods (e.g., fMRI, PET, ERP) require many trials to extract memory-related brain activity from ongoing brain activity (see Gazzaniga, Ivry, \& Mangun, 2002). Therefore, the goal of the present investigation was to compile an extensive database of gender-related and gender-neutral words that would allow researchers to select stimuli for almost any memory research application.

\section{METHOD}

\section{Participants}

The survey was completed by an initial sample of 177 undergraduates (56 males, 121 females) from the College of New Jersey. The results of this initial sample were compared with two additional samples of students from different geographic regions in the United States, to assess the generalizability of the results. Thus, a sample of 98 undergraduates ( 50 males, 48 females) attending North Carolina State University and 81 undergraduates $(24$ males, $57 \mathrm{fe}-$ males) attending the University of North Texas also completed the survey.

\section{Procedure}

Due to the lack of a preexisting database, a list of gender-related and gender-neutral words was developed on the basis of face validity and previous lists from the literature. Words in these lists were carefully selected to include various parts of speech (e.g., nouns, verbs, and adjectives). These items were pilot tested to confirm the researchers' initial assessment of the gender association of each word and to create word lists that were very similar in word frequency (Kučera \& Francis, 1967) and length. A list of 124 masculine (frequency, $M=38.00$ occurrences/million, range $=1-464$; length, $M=6.79$ letters, range $=3-12$ letters), 124 feminine (frequency, $M=25.07$, range $=1-232$; length, $M=7.00$, range = $3-13$ ), and 352 neutral (frequency, $M=47.85$, range $=1-442$; length, $M=6.14$, range $=3-12$ ) words was refined through several pilot-testing sessions. The formal survey used in this study was created by randomly ordering these items. The participants were informed that the purpose of this survey was to determine the gender association of a list of words. To complete the survey, the participants rated each item on a Likert Scale from 1 to $5(1=$ definitely feminine, $2=$ somewhat feminine, $3=$ neutral, $4=$ somewhat mas culine, and 5 = definitely masculine).

Although the participants were not instructed to determine the gender stereotypicality of each of the stimuli, gender relatedness can be inferred to coincide with gender stereotypicality. For example, by determining that a particular word (e.g., ballerina) is associated with a gender (female), the participant has positioned that particular stimulus under the mentally represented category of females. As many social cognitive psychologists have indicated, stereotypes can be understood as similar to other categorical generalizations (Insko \& Schopler, 1972). On the basis of this assumption, the authors agree with McCauley, Stitt, and Segal (1980) in defining a stereotype as "a name given to a generalization about a class of people." Adjectives correspond to personality traits (e.g., caring) shared by a particular gender, whereas behaviors (e.g., boxing) are reflected in verbs. Although nouns (e.g., daughter) may be by definition female, they can be thought of as gender stereotypical because the social roles to which individuals subscribe to are known to be generally associated with a particular gender (Diekman, Eagly, \& Kulesa, 2002). Because these behaviors, personality traits, and nouns can be associated with one gender more so than with another, they may be generally construed as gender stereotypical.

\section{RESULTS}

The mean and standard deviation word ratings were calculated for all 600 words. The values are presented in ascending order on the basis of the data obtained from the original sample collected at the College of New Jersey. The corresponding ratings from the other two samples are displayed in the adjacent columns in the tables. For ease of reference, we divided the entire list into three tables, which may be found in the Appendix. Table A1 presents words that have a feminine association (as defined by a mean rating of 1-2.49). Table A2 presents words that tend to be gender neutral (mean ratings of 2.5-3.49), and Table A3 presents words that have a masculine association (mean ratings of 3.5-5). For some of the words, the mean ratings varied considerably depending on the sample. These words are indicated in bold typeface in the tables. In addition to the mean ratings, the number of letters and word frequency (Kučera \& Francis, 1967) are presented for each item, to create a database that can be easily searched and reordered according to any attribute.

In addition to the general descriptive statistics, we analyzed the data in order to look for differences in item ratings as a function of the sex of the rater. Tables A4, A5, and A6 present the items that show an overall difference in classification by males and females. To determine these differences, word ratings by male and female participants were compared for significant differences, using $t$ tests for samples with uneven variances. Although this procedure revealed many statistical differences, we present only the items for which the mean overall group rating (presented in Tables A1-A3) might be misrepresentative, because there was inconsistency in the ratings across the different sexes. Thus, items were selected for Tables A4-A6 on the basis of a significant difference in the mean rating across the sexes and the working definition of gender categories used in this article. For example, an item was included in Tables A4A6 if the overall group rating was neutral (i.e., rated 2.50-3.49) and men tended to rate the word as neutral, whereas women tended to rate the word as masculine (i.e., >3.49).

Table A4 presents this subset of significant differences observed in the New Jersey sample, Table A5 presents the data observed in the North Carolina sample, and Table A6 presents the significant differences observed in the Texas data. When the word ratings from the New Jersey sample were subjected to the $t$ test analyses of mean ratings by the sex of the participant, significant differences were observed for 138 of the 600 items (or $23 \%$ ). However, these ratings were category consistent for all but 19 of the 138 items. Analyses of the North Carolina sample revealed significant differences for 36 of the 600 items $(6 \%)$, and the ratings were category consistent for all but 7 of the 36 items. Finally, analyses of the Texas sample revealed significant differences for 34 
of the 600 items $(6 \%)$, and the ratings were category consistent for all but 9 of the 36 items. This more detailed inspection of the analyses reveals that the vast majority of word ratings presented in our database have similar gender ratings for male and female participants.

Of course, there are many more differences between ratings of the sexes that may be of interest to others. For example, a subset of the items for which there is a half point or greater difference between male raters and female raters could be derived. As a result, we invite other scientists to explore these (and other) effects in the data themselves. ${ }^{1}$

\section{DISCUSSION}

The purpose of this survey was to develop a comprehensive list of masculine, feminine, and neutral words. The results of the survey identified approximately 60 words that have a strong masculine and feminine association, as well as a number of words that have a moderate association with one of the sexes. We also identified a substantial number of gender-neutral words, which can be used to select control words to match almost any research application. Because we collected data from three different regions of the United States, this list provides some assurances that the gender associations are not specific to one sample.

We believe that this list will serve as a valuable resource for researchers investigating gender stereotypes in memory. Because the words are listed according to their mean rating on the survey, a subset of words can be easily generated for almost any application. For example, if one wants to test masculine and feminine adjectives, these words can be quickly selected. Then the list of neutral words can be searched for gender-neutral adjectives. The extensive list of neutral words allows researchers to control for many extraneous variables, such as word frequency or length (which are also presented in the tables). In addition, one can examine memory for words that have a strong gender association (those at the extreme ends of the database) or can test memory for words that have a weaker gender association. In addition, if researchers wish to use stimuli that found much agreement among raters, items with small standard deviations can be selected. By providing a long list of words with and without gender associations, these stimulus materials can be applied to technologies that require a large number of observations (i.e., brain-imaging technology). ${ }^{2}$

We believe that this list will serve as a valuable resource not only for social cognitive psychologists investigating memory, but also for researchers in various psychological disciplines. For example, these words could be used by social psychologists in studies of gendered attitude assessment. The Bem Sex-Role Inventory (Bem, 1974) was developed to assess levels of individuals' an- drogyny. The list generated by our research could add breadth to the Bem Sex-Role Inventory and could be further refined for other clinical assessments related to gender identity and personality. Edelbrock and Sugawara (1978) and Kail and Levine (1976) created very limited pools of gender-specific traits as assessed by young children. In a more expansive fashion, by administering the list presented in this article to different age groups, developmental psychologists could broaden their understanding of the development of gender stereotyping.

Although the present investigation makes an important advance in identifying gender stereotypic and genderneutral words, future research could improve upon the areas in which this present study was limited. For instance, ratings were carefully collected from three distinctive regions of the United States; however, they cannot represent the gender-stereotypical beliefs of people from different cultures or even subcultures in the United States. In addition, gender roles and norms have changed dramatically in the United States over the past century. Further erosion of the differences between men and women in the workplace and at home may alter the gender ratings of the stimuli over time. The data are also limited in that they represent the beliefs of college-aged individuals, and we suspect that gender-relatedness ratings will vary considerably by age. Despite these limitations, this research will most certainly provide a tool that researchers in various fields might utilize to further our knowledge of the influence of gender on cognition.

\section{REFERENCES}

Allport, G. W. (1954). The nature of prejudice. Oxford: AddisonWesley.

Baddeley, A. D., Thomson, N., \& Buchanan, M. (1975). Word length and the structure of short-term memory. Journal of Verbal Learning \& Verbal Behavior, 14, 575-589.

Banaji, M. R., \& Greenwald, A. G. (1995). Implicit gender stereotyping in judgments of fame. Journal of Personality \& Social Psychology, 68, 181-198.

BEM, S. L. (1974). The measurement of psychological androgyny. Journal of Consulting \& Clinical Psychology, 42, 155-162.

Bessenoff, G. R., \& Sherman, J. W. (2000). Automatic and controlled components of prejudice toward fat people: Evaluation versus stereotype activation. Social Cognition, 18, 329-353.

Blair, I. V., \& BanaJI, M. R. (1996). Automatic and controlled processes in stereotype priming. Journal of Personality \& Social Psychology, 70, 1142-1163.

Carreiras, M., Garnham, A., OAkhill, J., \& Cain, K. (1996). The use of stereotypic gender information in constructing a mental model: Evidence from English and Spanish. Quarterly Journal of Experimental Psychology, 49A, 639-663.

DeEse, J. (1961). From the isolated verbal unit to connected discourse. In C. N. Cofer (Ed.), Verbal learning and verbal behavior (pp. 1131). New York: McGraw-Hill.

Diekman, A. B., Eagly, A. H., \& Kulesa, P. (2002). Accuracy and bias in stereotypes about the social and political attitudes of women and men. Journal of Experimental Social Psychology, 38, 268-282.

Edelbrock, C., \& Sugawara, A. I. (1978). Acquisition of sex-typed preferences in preschool-aged children. Developmental Psychology, 14, 614-623. 
Gazzaniga, M. S., Ivry, R. B., \& Mangun, G. R. (2002). Fundamentals of cognitive neuroscience (2nd ed.). New York: Norton.

Greenwald, A. G., \& BanaJi, M. R. (1995). Implicit social cognition: Attitudes, self-esteem, and stereotypes. Psychological Review, 102, 4-27.

InsKo, C. A., \& Schopler, J. (1972). Experimental social psychology. New York: Academic Press.

KaIL, R. V., \& LeVINE, L. E. (1976). Encoding processes and sex-role preferences. Journal of Experimental Child Psychology, 21, 256-263.

KATZ, D., \& BRALY, K. (1933). Racial stereotypes of one hundred college students. Journal of Abnormal \& Social Psychology, 28, 280190

KUČERA, H., \& FRANCIS, W. N. (1967). Computational analysis of presentday American English. Providence, RI: Brown University Press.

Macrae, C. N., Alnwick, K. A., Milne, A. B., \& Schloerscheidt, A. M. (2002). Person perception across the menstrual cycle: Hormonal influences on social-cognitive functioning. Psychological Science, 13, 532-536.

Macrae, C. N., Mitchell, J. P., \& Pendry, L. F. (2002). What's in a forename? Cue familiarity and stereotypical thinking. Journal of Experimental Social Psychology, 38, 186-193.

Mather, M., Johnson, M. K., \& De Leonardis, D. M. (1999). Stereotype reliance in source monitoring: Age differences and neuropsychological test correlates. Cognitive Neuropsychology, 16, 437-458.

McCauley, C., Stitt, C. L., \& Segal, M. (1980). Stereotyping: From prejudice to prediction. Psychological Bulletin, 87, 195-208.

PAIVIO, A. (1969). Mental imagery in associative learning and memory. Psychological Review, 76, 241-263.

Rosenkrantz, P., Vogel, S., Bee, H., Broverman, I., \& Broverman, D. M. (1968). Sex-role stereotypes and self-concepts in college students. Journal of Consulting \& Clinical Psychology, 32, 287-295.

SCHNEIDER, D. J. (1996). Modern stereotype research: Unfinished business. In C. N. Macrae, C. Stangor, \& M. Hewstone (Eds.), Stereotypes and stereotyping (pp. 419-453). New York: Guilford.

\section{NOTES}

1. Readers interested in obtaining an electronic version of the raw SPSS datafile can access this file via the Psychonomic Society Web archive. See the "Archived Materials" instructions at the end of this article.

2. Readers interested in obtaining an electronic version of the Microsoft Excel file containing mean ratings, standard deviations, number of letters, and word frequency for each stimulus can access this file via the Psychonomic Society Web archive. See the "Archived Materials" instructions at the end of this article.

\section{ARCHIVED MATERIALS}

The following materials and links may be accessed through the Psychonomic Society's Norms, Stimuli, and Data archive, http://www.psychonomic.org/archive/.

To access these files or links, search the archive for this article using the journal (Behavior Research Methods, Instruments, \& Computers), the first author's name (Crawford), and the publication year (2004).

FILE: Crawford-BRMIC-2004.zip

DESCRIPTION: The compressed archive file contains two files:

crawford2004tables1_3.xls, containing the word lists presented in Tables 1-3 of this article in a file generated by Excel 2002, which can be sorted according to any variable. Each row in the file represents one of the 600 stimulus words; each column, one of the dependent measures (mean and $S D$ for each sample, number of letters, frequency).

crawford2004raw_data.sav, containing the raw data analyzed in this article in a file generated by SPSS 11.0.1. Each row in the file represents a case (participant's ratings) for each of the 600 stimulus words; columns contain variables for sample location (New Jersey, North Carolina, or Texas), sample identification number, subject identification number, sex of the participant ( $1=$ male, $2=$ female $)$, and rating assigned to each stimulus word (described in the article).

AUTHOR's E-MAIL ADDRESS: jarretc@eden.rutgers.edu. 
APPENDIX

\begin{tabular}{|c|c|c|c|c|c|c|c|c|}
\hline \multicolumn{9}{|c|}{$\begin{array}{c}\text { Table A1 } \\
\text { Feminine Words (1-2.49) }\end{array}$} \\
\hline \multirow[b]{2}{*}{ Word } & \multicolumn{2}{|c|}{$\mathrm{NJ}$} & \multicolumn{2}{|c|}{$\mathrm{NC}$} & \multicolumn{2}{|c|}{$\mathrm{TX}$} & \multirow{2}{*}{$\begin{array}{l}\text { No. of } \\
\text { Letters }\end{array}$} & \multirow[b]{2}{*}{ Frequency } \\
\hline & $M$ & $S D$ & $M$ & $S D$ & $M$ & $S D$ & & \\
\hline Wife & 1.022 & 0.237 & 1.020 & 0.142 & 1.037 & 0.190 & 4 & 228 \\
\hline Princess & 1.028 & 0.166 & 1.082 & 0.469 & 1.062 & 0.242 & 8 & 10 \\
\hline Bridesmaid & 1.034 & 0.236 & 1.031 & 0.225 & 1.049 & 0.312 & 10 & 1 \\
\hline Pregnant & 1.039 & 0.326 & 1.061 & 0.280 & 1.111 & 0.353 & 8 & 8 \\
\hline Bride & 1.045 & 0.278 & 1.020 & 0.142 & 1.086 & 0.360 & 5 & 33 \\
\hline Grandmother & 1.050 & 0.220 & 1.061 & 0.241 & 1.160 & 0.460 & 11 & 9 \\
\hline Aunt & 1.051 & 0.287 & 1.020 & 0.142 & 1.099 & 0.436 & 4 & 22 \\
\hline Mother & 1.073 & 0.464 & 1.010 & 0.101 & 1.099 & 0.339 & 6 & 216 \\
\hline Bikini & 1.073 & 0.302 & 1.041 & 0.199 & 1.197 & 0.621 & 6 & 1 \\
\hline Daughter & 1.079 & 0.344 & 1.051 & 0.221 & 1.136 & 0.440 & 8 & 72 \\
\hline Dress & 1.079 & 0.271 & 1.112 & 0.317 & 1.185 & 0.503 & 5 & 67 \\
\hline Queen & 1.079 & 0.376 & 1.112 & 0.348 & 1.235 & 0.729 & 5 & 41 \\
\hline Sister & 1.101 & 0.440 & 1.122 & 0.413 & 1.136 & 0.379 & 6 & 38 \\
\hline Sorority & 1.118 & 0.416 & 1.163 & 0.550 & 1.309 & 0.701 & 8 & 1 \\
\hline Pantyhose & 1.124 & 0.448 & 1.153 & 0.415 & 1.210 & 0.607 & 9 & 1 \\
\hline Nun & 1.124 & 0.421 & 1.194 & 0.511 & 1.333 & 0.671 & 3 & 2 \\
\hline Lingerie & 1.130 & 0.452 & 1.194 & 0.446 & 1.222 & 0.548 & 8 & 2 \\
\hline Ovulate & 1.135 & 0.481 & 1.175 & 0.457 & 1.235 & 0.554 & 7 & 1 \\
\hline Maiden & 1.146 & 0.370 & 1.204 & 0.405 & 1.197 & 0.459 & 6 & 2 \\
\hline Menstruation & 1.146 & 0.544 & 1.296 & 0.692 & 1.309 & 0.752 & 12 & 1 \\
\hline Lipstick & 1.169 & 0.445 & 1.224 & 0.528 & 1.210 & 0.410 & 8 & 3 \\
\hline Mascara & 1.182 & 0.502 & 1.306 & 0.616 & 1.235 & 0.531 & 7 & 1 \\
\hline Niece & 1.192 & 0.448 & 1.143 & 0.352 & 1.284 & 0.617 & 5 & 8 \\
\hline Heiress & 1.194 & 0.499 & 1.234 & 0.639 & 1.284 & 0.617 & 7 & 1 \\
\hline Barbie & 1.197 & 0.500 & 1.133 & 0.370 & 1.296 & 0.621 & 6 & 1 \\
\hline Maternal & 1.198 & 0.544 & 1.378 & 0.711 & 1.444 & 0.837 & 8 & 5 \\
\hline Garter & 1.215 & 0.563 & 1.316 & 0.636 & 1.876 & 1.239 & 6 & 2 \\
\hline Estrogen & 1.220 & 0.667 & 1.347 & 0.875 & 1.284 & 0.675 & 8 & 1 \\
\hline Mistress & 1.231 & 0.561 & 1.204 & 0.453 & 1.333 & 0.707 & 8 & 5 \\
\hline Ballerina & 1.233 & 0.498 & 1.316 & 0.489 & 1.358 & 0.712 & 9 & 1 \\
\hline Cosmetic & 1.237 & 0.500 & 1.255 & 0.504 & 1.346 & 0.616 & 8 & 1 \\
\hline Damsel & 1.243 & 0.546 & 1.290 & 0.558 & 1.432 & 0.741 & 6 & 1 \\
\hline Doll & 1.260 & 0.500 & 1.204 & 0.405 & 1.333 & 0.775 & 4 & 11 \\
\hline Makeup & 1.260 & 0.586 & 1.247 & 0.457 & 1.309 & 0.539 & 6 & 1 \\
\hline Gown & 1.266 & 0.651 & 1.398 & 0.700 & 1.370 & 0.697 & 4 & 16 \\
\hline Duchess & 1.293 & 0.606 & 1.347 & 0.594 & 1.358 & 0.747 & 7 & 1 \\
\hline Dollhouse & 1.299 & 0.528 & 1.357 & 0.523 & 1.444 & 0.689 & 9 & 1 \\
\hline Skirt & 1.305 & 0.655 & 1.306 & 0.506 & 1.383 & 0.699 & 5 & 21 \\
\hline Stewardess & 1.339 & 0.629 & 1.571 & 0.746 & 1.469 & 0.792 & 10 & 2 \\
\hline Bitch & 1.355 & 0.668 & 1.398 & 0.700 & 1.605 & 0.944 & 5 & 6 \\
\hline Prostitute & 1.361 & 0.607 & 1.388 & 0.603 & 1.617 & 0.768 & 10 & 6 \\
\hline Ponytail & 1.372 & 0.636 & 1.408 & 0.686 & 1.395 & 0.719 & 8 & 1 \\
\hline Pink & 1.379 & 0.552 & 1.316 & 0.585 & 1.395 & 0.736 & 4 & 48 \\
\hline Nanny & 1.379 & 0.542 & 1.306 & 0.545 & 1.469 & 0.776 & 5 & 1 \\
\hline Manicure & 1.384 & 0.532 & 1.459 & 0.691 & 1.593 & 0.667 & 8 & 1 \\
\hline Blouse & 1.389 & 0.565 & 1.398 & 0.570 & 1.432 & 0.723 & 6 & 1 \\
\hline Perfume & 1.401 & 0.586 & 1.454 & 0.677 & 1.383 & 0.699 & 7 & 10 \\
\hline Ballet & 1.401 & 0.577 & 1.520 & 0.630 & 1.506 & 0.744 & 4 & 45 \\
\hline Pretty & 1.412 & 0.516 & 1.571 & 0.556 & 1.457 & 0.571 & 6 & 109 \\
\hline Beautiful & 1.418 & 0.589 & 1.449 & 0.628 & 1.469 & 0.691 & 9 & 127 \\
\hline Bouquet & 1.418 & 0.617 & 1.429 & 0.556 & 1.568 & 0.706 & 7 & 4 \\
\hline Necklace & 1.441 & 0.647 & 1.480 & 0.630 & 1.531 & 0.691 & 8 & 3 \\
\hline Petite & 1.452 & 0.573 & 1.582 & 0.591 & 1.494 & 0.709 & 6 & 1 \\
\hline Earrings & 1.452 & 0.682 & 1.388 & 0.683 & 1.383 & 0.624 & 8 & 3 \\
\hline Witches & 1.457 & 0.639 & 1.490 & 0.579 & 1.469 & 0.672 & 7 & 8 \\
\hline Fairy & 1.457 & 0.612 & 1.653 & 0.690 & 1.654 & 0.839 & 5 & 4 \\
\hline Corset & 1.466 & 0.820 & 1.694 & 0.913 & 1.370 & 0.601 & 6 & 1 \\
\hline Voluptuous & 1.475 & 0.683 & 1.615 & 0.800 & 1.506 & 0.744 & 10 & 3 \\
\hline Bonnet & 1.502 & 0.640 & 1.602 & 0.669 & 1.531 & 0.709 & 6 & 3 \\
\hline Pedicure & 1.519 & 0.707 & 1.735 & 0.767 & 1.617 & 0.699 & 8 & 1 \\
\hline Flowers & 1.531 & 0.682 & 1.633 & 0.738 & 1.568 & 0.741 & 7 & 57 \\
\hline Purse & 1.531 & 0.769 & 1.469 & 0.677 & 1.518 & 0.792 & 5 & 14 \\
\hline Blossom & 1.548 & 0.673 & 1.520 & 0.677 & 1.518 & 0.709 & 7 & 7 \\
\hline Anorexia & 1.553 & 0.664 & 1.735 & 0.682 & 1.790 & 0.754 & 8 & 1 \\
\hline Barrette & 1.581 & 1.025 & 1.816 & 0.976 & 1.605 & 0.970 & 8 & 1 \\
\hline Secretary & 1.593 & 0.624 & 1.633 & 0.648 & 1.580 & 0.788 & 9 & 191 \\
\hline
\end{tabular}


APPENDIX (Continued)

Table A1 (Continued)

\begin{tabular}{|c|c|c|c|c|c|c|c|c|}
\hline \multirow[b]{2}{*}{ Word } & \multicolumn{2}{|c|}{ NJ } & \multicolumn{2}{|c|}{$\mathrm{NC}$} & \multicolumn{2}{|c|}{ TX } & \multirow{2}{*}{$\begin{array}{l}\text { No. of } \\
\text { Letters }\end{array}$} & \multirow[b]{2}{*}{ Frequency } \\
\hline & $M$ & $S D$ & $M$ & $S D$ & $M$ & $S D$ & & \\
\hline Knitting & 1.598 & 0.701 & 1.683 & 0.683 & 1.716 & 0.855 & 8 & 1 \\
\hline Babysitter & 1.604 & 0.613 & 1.653 & 0.611 & 1.728 & 0.707 & 10 & 1 \\
\hline Corsage & 1.610 & 0.983 & 1.786 & 1.096 & 1.753 & 1.124 & 7 & 1 \\
\hline Nurse & 1.644 & 0.605 & 1.571 & 0.642 & 1.716 & 0.693 & 5 & 17 \\
\hline Bracelet & 1.649 & 0.666 & 1.704 & 0.661 & 1.667 & 0.689 & 8 & 1 \\
\hline Virginal & 1.666 & 0.758 & 1.633 & 0.709 & 1.728 & 0.922 & 8 & 1 \\
\hline Librarian & 1.700 & 0.703 & 1.878 & 0.790 & 1.827 & 0.905 & 9 & 5 \\
\hline Needlepoint & 1.717 & 0.790 & 1.888 & 0.811 & 1.765 & 0.912 & 11 & 1 \\
\hline Rose & 1.723 & 0.736 & 1.826 & 0.704 & 1.876 & 0.764 & 4 & 86 \\
\hline Housekeeper & 1.723 & 0.663 & 1.837 & 0.714 & 1.704 & 0.813 & 11 & 2 \\
\hline Sewing & 1.779 & 0.740 & 1.755 & 0.643 & 1.753 & 0.767 & 6 & 10 \\
\hline Gossip & 1.819 & 0.731 & 1.826 & 0.718 & 1.802 & 0.781 & 6 & 13 \\
\hline Quilting & 1.870 & 0.730 & 1.959 & 0.772 & 1.802 & 0.872 & 8 & 1 \\
\hline Flirtatious & 1.904 & 0.781 & 1.980 & 0.746 & 2.074 & 0.932 & 11 & 1 \\
\hline Sensitive & 1.932 & 0.856 & 1.939 & 0.823 & 1.839 & 0.766 & 9 & 59 \\
\hline Rainbow & 1.988 & 0.775 & 2.153 & 0.791 & 1.988 & 0.901 & 7 & 4 \\
\hline Diet & 1.994 & 0.734 & 2.194 & 0.769 & 2.000 & 0.806 & 4 & 21 \\
\hline Silk & 2.005 & 0.764 & 2.031 & 0.710 & 1.876 & 0.797 & 4 & 12 \\
\hline Romantic & 2.022 & 0.790 & 2.122 & 0.763 & 1.988 & 0.829 & 8 & 32 \\
\hline Bunny & 2.028 & 0.751 & 2.041 & 0.731 & 1.938 & 0.827 & 5 & 1 \\
\hline Kitten & 2.039 & 0.668 & 1.929 & 0.790 & 2.074 & 0.803 & 6 & 5 \\
\hline Hopscotch & 2.045 & 0.774 & 2.347 & 0.719 & 2.296 & 0.715 & 9 & 1 \\
\hline Teacher & 2.067 & 0.794 & 2.092 & 0.719 & 2.062 & 0.856 & 7 & 80 \\
\hline Sweet & 2.073 & 0.715 & 2.102 & 0.634 & 1.963 & 0.797 & 5 & 70 \\
\hline Thin & 2.073 & 0.818 & 2.102 & 0.739 & 2.037 & 0.914 & 4 & 92 \\
\hline Housework & 2.084 & 0.721 & 1.888 & 0.744 & 2.086 & 0.869 & 9 & 2 \\
\hline Sentimental & 2.101 & 0.791 & 2.337 & 0.673 & 2.111 & 0.742 & 11 & 15 \\
\hline Love & 2.101 & 0.826 & 2.214 & 0.828 & 2.086 & 0.925 & 4 & 232 \\
\hline Jumprope & 2.124 & 0.773 & 2.439 & 0.733 & 2.494 & 0.727 & 8 & 1 \\
\hline Emotional & 2.141 & 0.736 & 2.214 & 0.596 & 2.000 & 0.742 & 9 & 68 \\
\hline Dance & 2.146 & 0.723 & 2.316 & 0.619 & 2.222 & 0.742 & 5 & 90 \\
\hline Innocent & 2.146 & 0.754 & 2.194 & 0.668 & 2.074 & 0.771 & 8 & 37 \\
\hline Hemming & 2.153 & 0.810 & 2.122 & 0.744 & 2.086 & 0.883 & 7 & 1 \\
\hline Caring & 2.163 & 0.631 & 2.306 & 0.633 & 2.000 & 0.790 & 6 & 10 \\
\hline Cooking & 2.169 & 0.772 & 2.153 & 0.664 & 2.025 & 0.894 & 7 & 32 \\
\hline Cry & 2.192 & 0.751 & 2.347 & 0.627 & 2.173 & 0.721 & 3 & 48 \\
\hline Gentle & 2.197 & 0.776 & 2.275 & 0.670 & 2.173 & 0.787 & 6 & 27 \\
\hline Nagging & 2.226 & 0.702 & 2.316 & 0.754 & 2.185 & 0.853 & 7 & 9 \\
\hline Sympathetic & 2.248 & 0.695 & 2.408 & 0.623 & 2.136 & 0.703 & 11 & 35 \\
\hline Laundry & 2.265 & 0.709 & 2.408 & 0.655 & 2.383 & 0.874 & 7 & 5 \\
\hline Garden & 2.271 & 0.718 & 2.337 & 0.717 & 2.111 & 0.742 & 6 & 60 \\
\hline Skinny & 2.293 & 0.800 & 2.388 & 0.698 & 2.259 & 0.803 & 6 & 9 \\
\hline Supportive & 2.301 & 0.759 & 2.418 & 0.759 & 2.099 & 0.831 & 10 & 7 \\
\hline Springtime & 2.395 & 0.658 & 2.541 & 0.595 & 2.309 & 0.785 & 10 & 4 \\
\hline Tango & 2.418 & 0.635 & 2.582 & 0.591 & 2.593 & 0.803 & 5 & 2 \\
\hline Desire & 2.435 & 0.728 & 2.531 & 0.735 & 2.407 & 0.946 & 6 & 79 \\
\hline Heaven & 2.440 & 0.721 & 2.622 & 0.618 & 2.407 & 0.803 & 6 & 43 \\
\hline Champagne & 2.446 & 0.745 & 2.582 & 0.759 & 2.346 & 0.809 & 9 & 13 \\
\hline Oven & 2.480 & 0.731 & 2.622 & 0.650 & 2.506 & 0.760 & 4 & 7 \\
\hline Grocery & 2.452 & 0.681 & 2.510 & 0.678 & 2.506 & 0.744 & 7 & 9 \\
\hline Patient & 2.457 & 0.730 & 2.571 & 0.673 & 2.346 & 0.824 & 7 & 86 \\
\hline Star & 2.457 & 0.722 & 2.510 & 0.707 & 2.346 & 0.824 & 4 & 25 \\
\hline Understanding & 2.463 & 0.612 & 2.633 & 0.632 & 2.481 & 0.808 & 13 & 121 \\
\hline
\end{tabular}

Table A2

Neutral Words $(2.5-3.49)$

\begin{tabular}{|c|c|c|c|c|c|c|c|c|}
\hline \multirow[b]{2}{*}{ Word } & \multicolumn{2}{|c|}{ NJ } & \multicolumn{2}{|c|}{$\mathrm{NC}$} & \multicolumn{2}{|c|}{$\mathrm{TX}$} & \multirow{2}{*}{$\begin{array}{l}\text { No. of } \\
\text { Letters }\end{array}$} & \multirow[b]{2}{*}{ Frequency } \\
\hline & $M$ & $S D$ & $M$ & $S D$ & $M$ & $S D$ & & \\
\hline Pleasing & 2.502 & 0.716 & 2.490 & 0.646 & 2.432 & 0.757 & 8 & 10 \\
\hline Trend & 2.502 & 0.700 & 2.704 & 0.578 & 2.593 & 0.648 & 5 & 46 \\
\hline Faithful & 2.508 & 0.666 & 2.663 & 0.517 & 2.309 & 0.831 & 8 & 12 \\
\hline Insecure & 2.525 & 0.657 & 2.551 & 0.705 & 2.321 & 0.803 & 8 & 3 \\
\hline Hope & 2.542 & 0.621 & 2.714 & 0.556 & 2.407 & 0.738 & 4 & 178 \\
\hline Fruit & 2.548 & 0.638 & 2.551 & 0.594 & 2.420 & 0.788 & 5 & 35 \\
\hline
\end{tabular}


APPENDIX (Continued)

\begin{tabular}{|c|c|c|c|c|c|c|c|c|}
\hline \multicolumn{9}{|c|}{ Table A2 (Continued) } \\
\hline \multirow[b]{2}{*}{ Word } & \multicolumn{2}{|c|}{$\mathrm{NJ}$} & \multicolumn{2}{|c|}{$\mathrm{NC}$} & \multicolumn{2}{|c|}{ TX } & \multirow{2}{*}{$\begin{array}{l}\text { No. of } \\
\text { Letters }\end{array}$} & \multirow[b]{2}{*}{ Frequency } \\
\hline & $M$ & $S D$ & $M$ & $S D$ & $M$ & $S D$ & & \\
\hline Passive & 2.553 & 0.681 & 2.714 & 0.658 & 2.543 & 0.807 & 7 & 11 \\
\hline Beach & 2.565 & 0.619 & 2.582 & 0.687 & 2.642 & 0.695 & 5 & 61 \\
\hline Wine & 2.576 & 0.653 & 2.612 & 0.713 & 2.543 & 0.822 & 4 & 72 \\
\hline Stove & 2.581 & 0.750 & 2.571 & 0.673 & 2.518 & 0.743 & 5 & 15 \\
\hline Color & 2.598 & 0.623 & 2.673 & 0.654 & 2.494 & 0.727 & 5 & 141 \\
\hline Eggs & 2.604 & 0.716 & 2.592 & 0.655 & 2.506 & 0.853 & 4 & 35 \\
\hline Share & 2.604 & 0.585 & 2.714 & 0.537 & 2.370 & 0.660 & 5 & 98 \\
\hline Spirit & 2.610 & 0.584 & 2.735 & 0.509 & 2.518 & 0.743 & 6 & 182 \\
\hline Island & 2.625 & 0.681 & 2.724 & 0.570 & 2.667 & 0.725 & 6 & 127 \\
\hline Warm & 2.627 & 0.580 & 2.796 & 0.591 & 2.654 & 0.809 & 4 & 67 \\
\hline Masquerade & 2.632 & 0.758 & 2.643 & 0.763 & 2.469 & 0.792 & 10 & 2 \\
\hline Broom & 2.632 & 0.765 & 2.653 & 0.761 & 2.457 & 0.867 & 5 & 2 \\
\hline Earth & 2.632 & 0.758 & 2.633 & 0.724 & 2.370 & 0.813 & 5 & 150 \\
\hline Grapefruit & 2.632 & 0.589 & 2.714 & 0.642 & 2.679 & 0.834 & 10 & 3 \\
\hline Gold & 2.644 & 0.785 & 2.857 & 0.760 & 3.000 & 0.962 & 4 & 52 \\
\hline Apple & 2.649 & 0.613 & 2.694 & 0.633 & 2.617 & 0.815 & 5 & 9 \\
\hline Shoes & 2.649 & 0.658 & 2.622 & 0.650 & 2.531 & 0.838 & 5 & 44 \\
\hline Bird & 2.683 & 0.575 & 2.724 & 0.639 & 2.617 & 0.644 & 4 & 31 \\
\hline Fine & 2.687 & 0.716 & 2.796 & 0.746 & 2.679 & 0.920 & 4 & 161 \\
\hline Acetone & 2.694 & 0.713 & 2.804 & 0.671 & 2.716 & 0.884 & 7 & 4 \\
\hline Happy & 2.706 & 0.526 & 2.684 & 0.585 & 2.469 & 0.760 & 5 & 98 \\
\hline Thanks & 2.711 & 0.585 & 2.816 & 0.462 & 2.580 & 0.589 & 6 & 37 \\
\hline Season & 2.711 & 0.585 & 2.734 & 0.651 & 2.543 & 0.791 & 6 & 105 \\
\hline Ethical & 2.717 & 0.639 & 2.806 & 0.568 & 2.765 & 0.694 & 7 & 29 \\
\hline Child & 2.723 & 0.561 & 2.775 & 0.487 & 2.642 & 0.638 & 5 & 213 \\
\hline Gallery & 2.723 & 0.561 & 2.765 & 0.514 & 2.593 & 0.755 & 7 & 31 \\
\hline Dependent & 2.727 & 0.704 & 2.663 & 0.608 & 2.691 & 0.785 & 9 & 40 \\
\hline Bumblebee & 2.728 & 0.635 & 2.878 & 0.723 & 2.625 & 0.603 & 9 & 2 \\
\hline Ripening & 2.733 & 0.547 & 2.755 & 0.575 & 2.667 & 0.725 & 8 & 3 \\
\hline Cotton & 2.734 & 0.641 & 2.735 & 0.548 & 2.790 & 0.754 & 6 & 38 \\
\hline Ramble & 2.740 & 0.648 & 2.857 & 0.609 & 2.654 & 0.761 & 6 & 3 \\
\hline Cordial & 2.744 & 0.791 & 2.875 & 0.653 & 2.556 & 0.724 & 7 & 6 \\
\hline Refine & 2.745 & 0.646 & 2.765 & 0.670 & 2.753 & 0.859 & 6 & 3 \\
\hline Butter & 2.751 & 0.635 & 2.694 & 0.526 & 2.617 & 0.699 & 6 & 27 \\
\hline Balloon & 2.757 & 0.586 & 2.663 & 0.555 & 2.630 & 0.797 & 7 & 10 \\
\hline Goose & 2.757 & 0.668 & 2.786 & 0.630 & 2.469 & 0.823 & 5 & 4 \\
\hline Moon & 2.768 & 0.600 & 2.786 & 0.646 & 2.506 & 0.709 & 4 & 60 \\
\hline Letter & 2.768 & 0.519 & 2.878 & 0.359 & 2.852 & 0.635 & 6 & 145 \\
\hline Fickle & 2.774 & 0.634 & 2.734 & 0.602 & 2.617 & 0.663 & 6 & 1 \\
\hline Parent & 2.785 & 0.563 & 2.816 & 0.484 & 2.802 & 0.660 & 6 & 15 \\
\hline Bag & 2.802 & 0.612 & 2.826 & 0.643 & 2.790 & 0.753 & 3 & 42 \\
\hline Piano & 2.807 & 0.619 & 2.694 & 0.633 & 2.630 & 0.766 & 5 & 38 \\
\hline Ocean & 2.807 & 0.530 & 2.775 & 0.682 & 2.691 & 0.831 & 5 & 34 \\
\hline Moderation & 2.813 & 0.481 & 2.878 & 0.413 & 2.667 & 0.612 & 10 & 3 \\
\hline Call & 2.819 & 0.512 & 2.929 & 0.437 & 2.679 & 0.629 & 4 & 188 \\
\hline Esteem & 2.819 & 0.640 & 2.796 & 0.555 & 2.679 & 0.704 & 6 & 5 \\
\hline Alone & 2.824 & 0.561 & 2.816 & 0.648 & 2.728 & 0.806 & 5 & 195 \\
\hline Decelerate & 2.829 & 0.580 & 2.867 & 0.510 & 2.864 & 0.787 & 10 & 1 \\
\hline Rain & 2.830 & 0.505 & 2.837 & 0.447 & 2.815 & 0.709 & 4 & 70 \\
\hline Water & 2.830 & 0.457 & 2.796 & 0.500 & 2.654 & 0.636 & 5 & 442 \\
\hline Market & 2.830 & 0.652 & 2.898 & 0.565 & 2.802 & 0.641 & 6 & 155 \\
\hline Articulate & 2.830 & 0.643 & 2.969 & 0.464 & 2.864 & 0.818 & 10 & 8 \\
\hline Modern & 2.836 & 0.523 & 2.816 & 0.563 & 2.753 & 0.750 & 6 & 198 \\
\hline Ideal & 2.840 & 0.593 & 2.878 & 0.387 & 2.876 & 0.578 & 5 & 61 \\
\hline Music & 2.841 & 0.591 & 2.929 & 0.437 & 2.914 & 0.674 & 5 & 216 \\
\hline Lake & 2.841 & 0.551 & 2.898 & 0.487 & 2.938 & 0.730 & 4 & 54 \\
\hline Maple & 2.841 & 0.497 & 2.888 & 0.474 & 2.765 & 0.746 & 5 & 7 \\
\hline Flip & 2.847 & 0.537 & 2.980 & 0.497 & 2.864 & 0.607 & 4 & 4 \\
\hline Impeccable & 2.847 & 0.634 & 2.949 & 0.598 & 2.839 & 0.782 & 10 & 6 \\
\hline Mouse & 2.853 & 0.674 & 2.918 & 0.511 & 2.802 & 0.697 & 5 & 10 \\
\hline Fabled & 2.853 & 0.533 & 2.827 & 0.477 & 2.753 & 0.643 & 6 & 4 \\
\hline Camera & 2.858 & 0.582 & 2.888 & 0.590 & 2.716 & 0.693 & 6 & 36 \\
\hline Sand & 2.858 & 0.461 & 2.949 & 0.462 & 2.815 & 0.527 & 4 & 28 \\
\hline Oxygen & 2.858 & 0.473 & 2.867 & 0.422 & 2.815 & 0.477 & 6 & 43 \\
\hline Mind & 2.858 & 0.519 & 2.888 & 0.377 & 2.839 & 0.661 & 4 & 325 \\
\hline
\end{tabular}


APPENDIX (Continued)

\begin{tabular}{|c|c|c|c|c|c|c|c|c|}
\hline \multicolumn{9}{|c|}{ Table A2 (Continued) } \\
\hline \multirow[b]{2}{*}{ Word } & \multicolumn{2}{|c|}{ NJ } & \multicolumn{2}{|c|}{$\mathrm{NC}$} & \multicolumn{2}{|c|}{$\mathrm{TX}$} & \multirow{2}{*}{$\begin{array}{l}\text { No. of } \\
\text { Letters }\end{array}$} & \multirow[b]{2}{*}{ Frequency } \\
\hline & $M$ & $S D$ & $M$ & $S D$ & $M$ & $S D$ & & \\
\hline Unison & 2.863 & 0.505 & 2.918 & 0.371 & 2.778 & 0.671 & 6 & 3 \\
\hline Aspirin & 2.864 & 0.567 & 2.857 & 0.537 & 2.839 & 0.715 & 7 & 3 \\
\hline Triangle & 2.864 & 0.547 & 2.888 & 0.535 & 2.802 & 0.714 & 8 & 4 \\
\hline Village & 2.864 & 0.615 & 2.939 & 0.534 & 2.963 & 0.679 & 7 & 72 \\
\hline Alphabet & 2.870 & 0.464 & 2.867 & 0.397 & 2.790 & 0.586 & 8 & 2 \\
\hline Grade & 2.870 & 0.426 & 2.878 & 0.413 & 2.914 & 0.552 & 5 & 35 \\
\hline Choice & 2.870 & 0.439 & 2.929 & 0.329 & 2.704 & 0.697 & 6 & 113 \\
\hline Glass & 2.870 & 0.522 & 2.888 & 0.515 & 2.827 & 0.667 & 5 & 99 \\
\hline Lukewarm & 2.870 & 0.583 & 3.031 & 0.464 & 2.963 & 0.621 & 8 & 5 \\
\hline Learn & 2.870 & 0.412 & 2.867 & 0.490 & 2.827 & 0.587 & 5 & 84 \\
\hline Health & 2.875 & 0.421 & 2.969 & 0.417 & 2.728 & 0.633 & 6 & 105 \\
\hline Feed & 2.875 & 0.580 & 2.918 & 0.447 & 2.852 & 0.726 & 4 & 123 \\
\hline Alive & 2.875 & 0.447 & 2.969 & 0.303 & 2.839 & 0.601 & 5 & 57 \\
\hline Conniving & 2.875 & 0.816 & 2.694 & 0.724 & 2.802 & 1.005 & 9 & 1 \\
\hline Adjective & 2.875 & 0.378 & 3.010 & 0.268 & 2.889 & 0.474 & 9 & 2 \\
\hline Maybe & 2.875 & 0.435 & 2.908 & 0.408 & 2.889 & 0.524 & 5 & 134 \\
\hline Complete & 2.881 & 0.491 & 2.949 & 0.462 & 2.889 & 0.592 & 8 & 181 \\
\hline Near & 2.881 & 0.357 & 2.898 & 0.366 & 2.827 & 0.543 & 4 & 198 \\
\hline Narrow & 2.887 & 0.498 & 2.878 & 0.482 & 2.889 & 0.758 & 6 & 63 \\
\hline Lettuce & 2.887 & 0.411 & 2.837 & 0.531 & 2.728 & 0.652 & 7 & 1 \\
\hline Leaf & 2.887 & 0.424 & 2.918 & 0.469 & 2.765 & 0.694 & 4 & 12 \\
\hline Octave & 2.887 & 0.592 & 2.867 & 0.490 & 2.988 & 0.680 & 6 & 2 \\
\hline Frame & 2.887 & 0.601 & 2.929 & 0.523 & 2.839 & 0.661 & 5 & 74 \\
\hline Vision & 2.887 & 0.531 & 2.959 & 0.404 & 2.839 & 0.661 & 6 & 56 \\
\hline Float & 2.887 & 0.475 & 2.908 & 0.324 & 2.827 & 0.667 & 5 & 3 \\
\hline Livelihood & 2.892 & 0.578 & 2.898 & 0.507 & 2.815 & 0.635 & 10 & 5 \\
\hline Sound & 2.892 & 0.361 & 2.969 & 0.335 & 2.901 & 0.374 & 5 & 204 \\
\hline Liquid & 2.892 & 0.458 & 2.980 & 0.320 & 2.839 & 0.697 & 6 & 48 \\
\hline Visit & 2.898 & 0.427 & 2.939 & 0.375 & 2.778 & 0.570 & 5 & 109 \\
\hline Notice & 2.898 & 0.427 & 2.990 & 0.393 & 2.827 & 0.703 & 6 & 59 \\
\hline Trickle & 2.898 & 0.574 & 2.847 & 0.544 & 2.654 & 0.655 & 7 & 2 \\
\hline Book & 2.904 & 0.570 & 2.980 & 0.574 & 2.988 & 0.716 & 4 & 193 \\
\hline Chair & 2.904 & 0.422 & 2.949 & 0.505 & 2.926 & 0.628 & 5 & 66 \\
\hline Noodle & 2.909 & 0.525 & 2.908 & 0.408 & 2.926 & 0.685 & 6 & 1 \\
\hline Reply & 2.909 & 0.480 & 2.939 & 0.346 & 2.750 & 0.585 & 5 & 42 \\
\hline Fastidious & 2.909 & 0.514 & 3.051 & 0.392 & 2.926 & 0.628 & 10 & 3 \\
\hline Observable & 2.909 & 0.503 & 3.000 & 0.454 & 2.790 & 0.564 & 10 & 3 \\
\hline Party & 2.909 & 0.659 & 3.061 & 0.553 & 3.062 & 0.658 & 5 & 216 \\
\hline Wind & 2.915 & 0.498 & 2.929 & 0.542 & 2.839 & 0.766 & 4 & 63 \\
\hline Surname & 2.915 & 0.970 & 3.010 & 1.010 & 3.296 & 1.123 & 7 & 3 \\
\hline Whichever & 2.920 & 0.483 & 2.969 & 0.441 & 3.012 & 0.559 & 9 & 6 \\
\hline Acorn & 2.920 & 0.482 & 2.908 & 0.539 & 2.802 & 0.679 & 6 & 1 \\
\hline Answer & 2.920 & 0.418 & 2.949 & 0.333 & 2.852 & 0.550 & 6 & 152 \\
\hline Price & 2.926 & 0.426 & 3.020 & 0.517 & 2.876 & 0.678 & 5 & 108 \\
\hline Tongue & 2.926 & 0.574 & 2.847 & 0.439 & 2.889 & 0.806 & 6 & 35 \\
\hline Daily & 2.932 & 0.393 & 2.908 & 0.324 & 2.963 & 0.697 & 5 & 122 \\
\hline Hill & 2.932 & 0.471 & 2.969 & 0.507 & 3.000 & 0.612 & 4 & 72 \\
\hline Ill & 2.932 & 0.434 & 2.959 & 0.284 & 2.914 & 0.479 & 3 & 39 \\
\hline Carpet & 2.932 & 0.644 & 2.888 & 0.590 & 3.037 & 0.766 & 6 & 13 \\
\hline Echo & 2.949 & 0.514 & 3.010 & 0.487 & 2.778 & 0.612 & 4 & 10 \\
\hline Handbook & 2.949 & 0.556 & 2.878 & 0.359 & 2.790 & 0.684 & 8 & 2 \\
\hline Admit & 2.954 & 0.541 & 2.939 & 0.589 & 2.790 & 0.665 & 5 & 37 \\
\hline Myth & 2.954 & 0.531 & 3.071 & 0.523 & 3.012 & 0.766 & 4 & 35 \\
\hline Doorbell & 2.954 & 0.509 & 2.929 & 0.460 & 2.914 & 0.656 & 8 & 2 \\
\hline Crumb & 2.954 & 0.462 & 3.000 & 0.287 & 3.037 & 0.641 & 5 & 3 \\
\hline Apart & 2.960 & 0.389 & 3.020 & 0.379 & 2.889 & 0.474 & 5 & 57 \\
\hline Coffee & 2.960 & 0.567 & 2.888 & 0.515 & 2.963 & 0.660 & 6 & 78 \\
\hline Town & 2.960 & 0.389 & 2.878 & 0.482 & 3.062 & 0.556 & 4 & 212 \\
\hline Half & 2.960 & 0.268 & 2.898 & 0.465 & 2.889 & 0.447 & 4 & 275 \\
\hline East & 2.960 & 0.431 & 2.878 & 0.482 & 2.938 & 0.533 & 4 & 183 \\
\hline Independent & 2.960 & 0.785 & 2.980 & 0.688 & 2.914 & 0.964 & 11 & 70 \\
\hline Reach & 2.965 & 0.352 & 2.990 & 0.176 & 2.988 & 0.487 & 5 & 106 \\
\hline Dozen & 2.966 & 0.450 & 2.990 & 0.366 & 2.876 & 0.659 & 5 & 52 \\
\hline Drugstore & 2.966 & 0.487 & 3.041 & 0.536 & 3.086 & 0.552 & 9 & 5 \\
\hline
\end{tabular}


APPENDIX (Continued)

\begin{tabular}{|c|c|c|c|c|c|c|c|c|}
\hline \multicolumn{9}{|c|}{ Table A2 (Continued) } \\
\hline \multirow[b]{2}{*}{ Word } & \multicolumn{2}{|c|}{ NJ } & \multicolumn{2}{|c|}{$\mathrm{NC}$} & \multicolumn{2}{|c|}{$\mathrm{TX}$} & \multirow{2}{*}{$\begin{array}{l}\text { No. of } \\
\text { Letters }\end{array}$} & \multirow[b]{2}{*}{ Frequency } \\
\hline & $M$ & $S D$ & $M$ & $S D$ & $M$ & $S D$ & & \\
\hline Coat & 2.966 & 0.542 & 3.112 & 0.554 & 3.160 & 0.679 & 4 & 43 \\
\hline Convection & 2.966 & 0.542 & 3.010 & 0.366 & 2.963 & 0.679 & 10 & 2 \\
\hline River & 2.966 & 0.487 & 2.990 & 0.583 & 2.926 & 0.721 & 5 & 165 \\
\hline Underside & 2.966 & 0.411 & 2.949 & 0.440 & 2.938 & 0.457 & 9 & 3 \\
\hline Heat & 2.971 & 0.643 & 3.061 & 0.606 & 3.173 & 0.877 & 4 & 97 \\
\hline Mute & 2.971 & 0.360 & 2.990 & 0.337 & 2.889 & 0.570 & 4 & 3 \\
\hline Lunch & 2.971 & 0.404 & 2.950 & 0.462 & 2.889 & 0.418 & 5 & 33 \\
\hline Magic & 2.971 & 0.718 & 3.031 & 0.633 & 3.111 & 0.822 & 5 & 37 \\
\hline Deaf & 2.971 & 0.457 & 3.041 & 0.349 & 3.123 & 0.600 & 4 & 12 \\
\hline Guess & 2.977 & 0.521 & 2.918 & 0.398 & 3.111 & 0.632 & 5 & 56 \\
\hline Lens & 2.977 & 0.438 & 2.898 & 0.393 & 2.901 & 0.583 & 4 & 12 \\
\hline Toothbrush & 2.977 & 0.451 & 3.000 & 0.352 & 3.012 & 0.462 & 10 & 6 \\
\hline Precede & 2.983 & 0.482 & 3.051 & 0.362 & 2.963 & 0.641 & 7 & 3 \\
\hline Media & 2.983 & 0.558 & 2.959 & 0.429 & 3.074 & 0.738 & 5 & 13 \\
\hline Cadence & 2.983 & 0.527 & 3.206 & 0.676 & 2.988 & 0.798 & 7 & 2 \\
\hline Aggies & 2.988 & 0.512 & 3.206 & 0.628 & 3.617 & 0.943 & 6 & 2 \\
\hline Astound & 2.988 & 0.426 & 3.041 & 0.349 & 2.975 & 0.591 & 7 & 1 \\
\hline Oncoming & 2.988 & 0.439 & 3.031 & 0.303 & 3.037 & 0.459 & 8 & 2 \\
\hline Agile & 2.994 & 0.794 & 3.184 & 0.723 & 2.864 & 0.862 & 5 & 2 \\
\hline Biopsy & 2.994 & 0.598 & 3.093 & 0.597 & 2.975 & 0.774 & 6 & 2 \\
\hline Grass & 2.994 & 0.482 & 2.969 & 0.527 & 2.988 & 0.698 & 5 & 53 \\
\hline Banner & 3.000 & 0.478 & 2.990 & 0.442 & 3.123 & 0.556 & 6 & 8 \\
\hline Hamster & 3.000 & 0.715 & 2.929 & 0.561 & 3.074 & 0.685 & 7 & 1 \\
\hline Refill & 3.000 & 0.398 & 3.020 & 0.430 & 2.951 & 0.669 & 6 & 3 \\
\hline Sediment & 3.000 & 0.583 & 3.010 & 0.487 & 3.000 & 0.758 & 8 & 3 \\
\hline Weekday & 3.000 & 0.319 & 3.071 & 0.437 & 3.160 & 0.580 & 7 & 2 \\
\hline Tarnish & 3.000 & 0.614 & 2.980 & 0.497 & 2.988 & 0.642 & 7 & 1 \\
\hline Schema & 3.000 & 0.488 & 2.980 & 0.406 & 2.864 & 0.518 & 6 & 3 \\
\hline Malaria & 3.000 & 0.543 & 3.093 & 0.458 & 3.037 & 0.732 & 7 & 3 \\
\hline Calamity & 3.005 & 0.635 & 3.031 & 0.467 & 2.716 & 0.762 & 8 & 4 \\
\hline Undercurrent & 3.005 & 0.376 & 2.990 & 0.418 & 3.062 & 0.509 & 12 & 3 \\
\hline Usual & 3.005 & 0.407 & 2.969 & 0.418 & 3.012 & 0.512 & 5 & 96 \\
\hline Airline & 3.005 & 0.458 & 2.959 & 0.474 & 3.136 & 0.720 & 7 & 2 \\
\hline Offer & 3.005 & 0.447 & 3.051 & 0.300 & 3.000 & 0.592 & 5 & 80 \\
\hline Token & 3.011 & 0.522 & 2.980 & 0.406 & 3.037 & 0.580 & 5 & 10 \\
\hline Tree & 3.011 & 0.488 & 3.010 & 0.487 & 2.938 & 0.639 & 4 & 59 \\
\hline Nightfall & 3.011 & 0.553 & 2.949 & 0.505 & 2.864 & 0.802 & 9 & 4 \\
\hline Award & 3.011 & 0.522 & 3.061 & 0.401 & 2.938 & 0.619 & 5 & 46 \\
\hline Noun & 3.011 & 0.301 & 2.990 & 0.418 & 2.852 & 0.635 & 4 & 1 \\
\hline Deep & 3.016 & 0.578 & 2.980 & 0.465 & 3.148 & 0.776 & 4 & 109 \\
\hline Shake & 3.016 & 0.538 & 2.939 & 0.494 & 2.988 & 0.642 & 5 & 17 \\
\hline Oat & 3.016 & 0.432 & 3.082 & 0.447 & 3.185 & 0.572 & 3 & 1 \\
\hline Nose & 3.016 & 0.405 & 2.888 & 0.572 & 2.926 & 0.587 & 4 & 60 \\
\hline Student & 3.016 & 0.271 & 3.031 & 0.225 & 3.037 & 0.459 & 7 & 130 \\
\hline Impact & 3.022 & 0.510 & 3.082 & 0.447 & 3.074 & 0.608 & 6 & 67 \\
\hline Hideaway & 3.022 & 0.521 & 3.031 & 0.486 & 3.000 & 0.707 & 8 & 1 \\
\hline Film & 3.022 & 0.553 & 3.000 & 0.454 & 3.000 & 0.652 & 4 & 96 \\
\hline Kiosk & 3.022 & 0.487 & 2.887 & 0.557 & 2.975 & 0.707 & 5 & 1 \\
\hline Freedom & 3.022 & 0.510 & 3.143 & 0.592 & 3.086 & 0.728 & 7 & 128 \\
\hline Poor & 3.022 & 0.425 & 3.010 & 0.337 & 3.025 & 0.569 & 4 & 113 \\
\hline Arthritis & 3.028 & 0.652 & 3.153 & 0.563 & 3.160 & 0.889 & 9 & 3 \\
\hline Obvious & 3.028 & 0.515 & 3.041 & 0.516 & 3.099 & 0.700 & 7 & 92 \\
\hline Unexplained & 3.033 & 0.542 & 3.061 & 0.450 & 3.210 & 0.684 & 11 & 4 \\
\hline Knee & 3.033 & 0.351 & 3.061 & 0.426 & 3.123 & 0.458 & 4 & 35 \\
\hline Diagram & 3.033 & 0.450 & 2.929 & 0.387 & 2.975 & 0.707 & 7 & 10 \\
\hline Pandemic & 3.034 & 0.511 & 2.980 & 0.320 & 3.012 & 0.512 & 8 & 2 \\
\hline Plaintive & 3.039 & 0.644 & 2.888 & 0.535 & 3.025 & 0.632 & 9 & 2 \\
\hline Bank & 3.039 & 0.444 & 3.041 & 0.516 & 3.099 & 0.718 & 4 & 83 \\
\hline Logical & 3.039 & 0.660 & 3.153 & 0.598 & 3.062 & 0.764 & 7 & 34 \\
\hline Trauma & 3.045 & 0.646 & 3.133 & 0.530 & 3.000 & 0.725 & 6 & 1 \\
\hline Lobe & 3.045 & 0.462 & 2.980 & 0.476 & 3.012 & 0.602 & 4 & 3 \\
\hline Porch & 3.045 & 0.591 & 2.959 & 0.536 & 3.025 & 0.707 & 5 & 43 \\
\hline Cold & 3.045 & 0.497 & 3.000 & 0.556 & 3.012 & 0.716 & 4 & 171 \\
\hline Zealous & 3.045 & 0.619 & 3.010 & 0.442 & 2.951 & 0.705 & 7 & 4 \\
\hline Hotel & 3.045 & 0.509 & 3.000 & 0.574 & 3.197 & 0.679 & 5 & 126 \\
\hline
\end{tabular}


APPENDIX (Continued)

\begin{tabular}{|c|c|c|c|c|c|c|c|c|}
\hline \multicolumn{9}{|c|}{ Table A2 (Continued) } \\
\hline \multirow[b]{2}{*}{ Word } & \multicolumn{2}{|c|}{ NJ } & \multicolumn{2}{|c|}{$\mathrm{NC}$} & \multicolumn{2}{|c|}{ TX } & \multirow{2}{*}{$\begin{array}{l}\text { No. of } \\
\text { Letters }\end{array}$} & \multirow[b]{2}{*}{ Frequency } \\
\hline & $M$ & $S D$ & $M$ & $S D$ & $M$ & $S D$ & & \\
\hline Tube & 3.045 & 0.486 & 2.980 & 0.379 & 3.086 & 0.636 & 4 & 31 \\
\hline Key & 3.050 & 0.467 & 3.031 & 0.365 & 3.037 & 0.486 & 3 & 88 \\
\hline Algae & 3.050 & 0.491 & 3.020 & 0.497 & 2.938 & 0.677 & 5 & 7 \\
\hline Estimation & 3.050 & 0.479 & 3.000 & 0.382 & 3.173 & 0.648 & 10 & 4 \\
\hline Ferment & 3.050 & 0.503 & 2.980 & 0.556 & 2.975 & 0.724 & 7 & 2 \\
\hline Undefined & 3.051 & 0.359 & 2.960 & 0.452 & 3.037 & 0.486 & 9 & 4 \\
\hline Disease & 3.056 & 0.408 & 3.082 & 0.447 & 3.160 & 0.697 & 7 & 53 \\
\hline Ability & 3.056 & 0.422 & 3.092 & 0.354 & 3.284 & 0.637 & 7 & 74 \\
\hline Obtain & 3.062 & 0.402 & 3.010 & 0.176 & 2.963 & 0.601 & 6 & 42 \\
\hline Volume & 3.062 & 0.478 & 3.092 & 0.433 & 3.000 & 0.612 & 6 & 135 \\
\hline Guilt & 3.062 & 0.700 & 3.112 & 0.495 & 2.938 & 0.812 & 5 & 33 \\
\hline Concur & 3.062 & 0.523 & 3.020 & 0.406 & 3.025 & 0.632 & 6 & 4 \\
\hline Lexicon & 3.062 & 0.512 & 2.979 & 0.250 & 3.012 & 0.559 & 7 & 2 \\
\hline Confident & 3.067 & 0.703 & 3.184 & 0.581 & 3.111 & 0.880 & 9 & 16 \\
\hline Windshield & 3.067 & 0.447 & 3.041 & 0.452 & 3.197 & 0.660 & 10 & 6 \\
\hline Reverted & 3.067 & 0.393 & 2.990 & 0.304 & 3.222 & 0.707 & 8 & 2 \\
\hline Catsup & 3.067 & 0.483 & 2.938 & 0.496 & 2.963 & 0.558 & 7 & 3 \\
\hline Uneven & 3.073 & 0.439 & 3.020 & 0.202 & 3.025 & 0.524 & 6 & 6 \\
\hline Affix & 3.073 & 0.464 & 2.990 & 0.337 & 3.012 & 0.559 & 5 & 1 \\
\hline Escape & 3.073 & 0.554 & 3.092 & 0.408 & 3.074 & 0.771 & 6 & 65 \\
\hline North & 3.079 & 0.377 & 3.020 & 0.430 & 3.197 & 0.660 & 5 & 206 \\
\hline Match & 3.079 & 0.445 & 3.010 & 0.442 & 2.951 & 0.522 & 5 & 41 \\
\hline Couch & 3.079 & 0.505 & 3.082 & 0.604 & 3.247 & 0.767 & 5 & 12 \\
\hline Commercial & 3.079 & 0.482 & 3.051 & 0.462 & 3.123 & 0.659 & 10 & 61 \\
\hline Regulating & 3.079 & 0.625 & 3.133 & 0.468 & 3.197 & 0.697 & 10 & 4 \\
\hline Eat & 3.079 & 0.527 & 3.000 & 0.518 & 3.123 & 0.620 & 3 & 61 \\
\hline Spasm & 3.079 & 0.537 & 3.082 & 0.491 & 3.049 & 0.669 & 5 & 3 \\
\hline Elbow & 3.084 & 0.486 & 3.010 & 0.442 & 3.136 & 0.720 & 5 & 10 \\
\hline Crazy & 3.084 & 0.620 & 3.206 & 0.576 & 3.197 & 0.732 & 5 & 34 \\
\hline Rode & 3.084 & 0.509 & 3.082 & 0.447 & 3.259 & 0.608 & 4 & 40 \\
\hline Dormant & 3.084 & 0.552 & 3.020 & 0.556 & 3.025 & 0.632 & 7 & 5 \\
\hline Lease & 3.084 & 0.396 & 3.061 & 0.472 & 3.062 & 0.533 & 5 & 10 \\
\hline Invalid & 3.084 & 0.396 & 3.041 & 0.378 & 3.136 & 0.565 & 7 & 7 \\
\hline Addition & 3.084 & 0.509 & 3.051 & 0.462 & 2.988 & 0.750 & 8 & 142 \\
\hline Funny & 3.090 & 0.576 & 3.102 & 0.527 & 3.099 & 0.700 & 5 & 41 \\
\hline Tasteless & 3.090 & 0.468 & 3.000 & 0.431 & 3.012 & 0.559 & 9 & 1 \\
\hline Magnet & 3.090 & 0.469 & 3.021 & 0.540 & 3.000 & 0.671 & 6 & 3 \\
\hline Bottle & 3.090 & 0.596 & 3.051 & 0.544 & 3.062 & 0.695 & 6 & 76 \\
\hline Aversion & 3.090 & 0.597 & 2.959 & 0.404 & 3.111 & 0.632 & 8 & 2 \\
\hline Floor & 3.090 & 0.373 & 3.010 & 0.306 & 3.160 & 0.432 & 5 & 158 \\
\hline Rebuttal & 3.096 & 0.529 & 3.031 & 0.564 & 3.185 & 0.691 & 8 & 2 \\
\hline Green & 3.096 & 0.580 & 3.112 & 0.590 & 3.086 & 0.825 & 5 & 116 \\
\hline Union & 3.096 & 0.654 & 3.031 & 0.766 & 3.074 & 0.755 & 5 & 182 \\
\hline Scan & 3.096 & 0.364 & 2.990 & 0.366 & 3.012 & 0.512 & 4 & 5 \\
\hline Table & 3.101 & 0.440 & 3.020 & 0.286 & 2.988 & 0.642 & 5 & 198 \\
\hline Fork & 3.101 & 0.594 & 2.980 & 0.430 & 3.099 & 0.700 & 4 & 14 \\
\hline Deliberate & 3.101 & 0.544 & 3.041 & 0.429 & 3.197 & 0.781 & 10 & 15 \\
\hline Enigmatic & 3.107 & 0.568 & 3.031 & 0.464 & 3.160 & 0.679 & 9 & 2 \\
\hline Absent & 3.107 & 0.391 & 3.092 & 0.354 & 3.185 & 0.615 & 6 & 28 \\
\hline Energy & 3.113 & 0.574 & 3.194 & 0.550 & 3.086 & 0.728 & 6 & 100 \\
\hline Hexagon & 3.113 & 0.437 & 3.133 & 0.490 & 3.210 & 0.586 & 7 & 1 \\
\hline Expertise & 3.113 & 0.542 & 3.214 & 0.460 & 3.259 & 0.587 & 9 & 3 \\
\hline Paper & 3.113 & 0.411 & 3.010 & 0.418 & 3.136 & 0.565 & 5 & 157 \\
\hline Deteriorated & 3.118 & 0.479 & 3.143 & 0.574 & 3.247 & 0.783 & 12 & 1 \\
\hline Ahead & 3.118 & 0.524 & 3.153 & 0.544 & 3.049 & 0.669 & 5 & 109 \\
\hline Title & 3.118 & 0.442 & 3.061 & 0.450 & 3.197 & 0.534 & 5 & 77 \\
\hline Orthographic & 3.118 & 0.491 & 3.186 & 0.527 & 3.358 & 0.658 & 12 & 3 \\
\hline Furlough & 3.118 & 0.514 & 3.102 & 0.393 & 3.333 & 0.707 & 8 & 2 \\
\hline Decisive & 3.124 & 0.727 & 3.184 & 0.632 & 3.099 & 0.889 & 8 & 19 \\
\hline Exit & 3.124 & 0.529 & 3.061 & 0.426 & 3.247 & 0.560 & 4 & 7 \\
\hline Kinetic & 3.125 & 0.485 & 3.071 & 0.412 & 3.037 & 0.621 & 7 & 8 \\
\hline Vote & 3.129 & 0.476 & 3.133 & 0.397 & 3.210 & 0.541 & 4 & 75 \\
\hline False & 3.129 & 0.488 & 3.051 & 0.333 & 3.086 & 0.552 & 5 & 29 \\
\hline Achieving & 3.129 & 0.639 & 3.184 & 0.525 & 3.086 & 0.711 & 9 & 15 \\
\hline Bottleneck & 3.129 & 0.682 & 3.092 & 0.594 & 3.321 & 0.788 & 10 & 2 \\
\hline
\end{tabular}


APPENDIX (Continued)

\begin{tabular}{|c|c|c|c|c|c|c|c|c|}
\hline \multicolumn{9}{|c|}{ Table A2 (Continued) } \\
\hline \multirow[b]{2}{*}{ Word } & \multicolumn{2}{|c|}{ NJ } & \multicolumn{2}{|c|}{$\mathrm{NC}$} & \multicolumn{2}{|c|}{ TX } & \multirow{2}{*}{$\begin{array}{l}\text { No. of } \\
\text { Letters }\end{array}$} & \multirow[b]{2}{*}{ Frequency } \\
\hline & $M$ & $S D$ & $M$ & $S D$ & $M$ & $S D$ & & \\
\hline Quarter & 3.129 & 0.476 & 2.959 & 0.406 & 3.111 & 0.548 & 7 & 34 \\
\hline Tattered & 3.129 & 0.583 & 3.173 & 0.557 & 3.025 & 0.689 & 8 & 5 \\
\hline Tails & 3.130 & 0.565 & 3.041 & 0.608 & 3.099 & 0.752 & 5 & 7 \\
\hline Litigant & 3.135 & 0.547 & 3.072 & 0.439 & 3.099 & 0.625 & 8 & 2 \\
\hline Oblivion & 3.135 & 0.557 & 3.020 & 0.476 & 3.049 & 0.669 & 8 & 2 \\
\hline Deficient & 3.135 & 0.597 & 3.020 & 0.379 & 3.136 & 0.720 & 9 & 3 \\
\hline Pepper & 3.135 & 0.615 & 3.214 & 0.542 & 3.235 & 0.576 & 6 & 13 \\
\hline Ground & 3.135 & 0.417 & 3.082 & 0.469 & 3.235 & 0.638 & 6 & 186 \\
\hline Discredit & 3.135 & 0.504 & 3.031 & 0.486 & 3.160 & 0.641 & 9 & 2 \\
\hline Quick & 3.146 & 0.554 & 3.143 & 0.556 & 3.100 & 0.648 & 5 & 68 \\
\hline Odd & 3.146 & 0.465 & 3.153 & 0.439 & 3.222 & 0.632 & 3 & 44 \\
\hline Horse & 3.146 & 0.723 & 3.041 & 0.672 & 3.173 & 0.703 & 5 & 117 \\
\hline Target & 3.152 & 0.537 & 3.184 & 0.462 & 3.049 & 0.757 & 6 & 45 \\
\hline Document & 3.158 & 0.436 & 3.041 & 0.404 & 3.086 & 0.596 & 8 & 13 \\
\hline Jacket & 3.158 & 0.486 & 3.122 & 0.542 & 3.111 & 0.689 & 6 & 33 \\
\hline Trilogy & 3.163 & 0.466 & 3.125 & 0.363 & 3.296 & 0.601 & 7 & 4 \\
\hline Shin & 3.163 & 0.534 & 3.000 & 0.556 & 3.210 & 0.646 & 4 & 3 \\
\hline Contributor & 3.163 & 0.575 & 3.143 & 0.431 & 3.210 & 0.754 & 11 & 2 \\
\hline Worse & 3.169 & 0.445 & 3.092 & 0.408 & 3.148 & 0.572 & 5 & 50 \\
\hline Watch & 3.169 & 0.588 & 3.235 & 0.514 & 3.160 & 0.679 & 5 & 81 \\
\hline Radio & 3.169 & 0.470 & 3.051 & 0.264 & 3.247 & 0.603 & 5 & 120 \\
\hline Addiction & 3.175 & 0.628 & 3.092 & 0.519 & 3.272 & 0.880 & 9 & 3 \\
\hline Airplane & 3.180 & 0.440 & 3.102 & 0.465 & 3.296 & 0.766 & 8 & 11 \\
\hline Export & 3.180 & 0.414 & 3.122 & 0.359 & 3.444 & 0.707 & 6 & 10 \\
\hline Carbon & 3.186 & 0.444 & 3.163 & 0.423 & 3.185 & 0.527 & 6 & 30 \\
\hline Bite & 3.186 & 0.558 & 3.173 & 0.557 & 3.272 & 0.689 & 4 & 10 \\
\hline Compost & 3.186 & 0.537 & 3.165 & 0.472 & 3.259 & 0.648 & 7 & 8 \\
\hline Pocket & 3.186 & 0.493 & 3.153 & 0.439 & 3.222 & 0.707 & 6 & 46 \\
\hline Dislodge & 3.192 & 0.530 & 3.153 & 0.434 & 3.173 & 0.628 & 8 & 2 \\
\hline Quake & 3.192 & 0.496 & 3.082 & 0.531 & 3.272 & 0.707 & 5 & 2 \\
\hline Capacitor & 3.192 & 0.540 & 3.194 & 0.490 & 3.309 & 0.584 & 9 & 2 \\
\hline Molecule & 3.197 & 0.554 & 3.051 & 0.484 & 3.185 & 0.594 & 8 & 6 \\
\hline Racket & 3.197 & 0.511 & 3.224 & 0.584 & 3.358 & 0.811 & 6 & 5 \\
\hline Unit & 3.197 & 0.522 & 3.020 & 0.351 & 3.160 & 0.641 & 4 & 103 \\
\hline Theory & 3.197 & 0.488 & 2.990 & 0.366 & 3.111 & 0.500 & 6 & 129 \\
\hline Death & 3.197 & 0.648 & 3.326 & 0.670 & 3.309 & 0.736 & 5 & 277 \\
\hline Biography & 3.197 & 0.522 & 3.092 & 0.499 & 3.136 & 0.754 & 9 & 13 \\
\hline News & 3.203 & 0.536 & 3.173 & 0.538 & 3.321 & 0.629 & 4 & 102 \\
\hline Provoke & 3.203 & 0.624 & 3.133 & 0.490 & 3.370 & 0.732 & 7 & 3 \\
\hline Error & 3.204 & 0.505 & 3.071 & 0.437 & 3.287 & 0.750 & 5 & 36 \\
\hline Paint & 3.205 & 0.645 & 3.163 & 0.569 & 3.197 & 0.765 & 5 & 37 \\
\hline Irrigate & 3.209 & 0.550 & 3.265 & 0.602 & 3.333 & 0.652 & 8 & 1 \\
\hline Canyons & 3.210 & 0.646 & 3.061 & 0.623 & 3.136 & 0.771 & 7 & 2 \\
\hline Rectangle & 3.226 & 0.482 & 3.122 & 0.413 & 3.197 & 0.600 & 9 & 4 \\
\hline Wage & 3.231 & 0.508 & 3.224 & 0.487 & 3.259 & 0.587 & 4 & 56 \\
\hline Assertive & 3.233 & 0.690 & 3.216 & 0.767 & 3.136 & 0.833 & 9 & 2 \\
\hline Kennel & 3.237 & 0.543 & 3.194 & 0.586 & 3.346 & 0.616 & 6 & 3 \\
\hline Rapid & 3.237 & 0.500 & 3.133 & 0.510 & 3.185 & 0.673 & 5 & 43 \\
\hline Coffin & 3.237 & 0.564 & 3.265 & 0.509 & 3.457 & 0.742 & 6 & 7 \\
\hline Humor & 3.242 & 0.535 & 3.142 & 0.476 & 3.247 & 0.603 & 5 & 47 \\
\hline Wreck & 3.242 & 0.586 & 3.326 & 0.533 & 3.370 & 0.887 & 5 & 8 \\
\hline Gripping & 3.248 & 0.617 & 3.275 & 0.493 & 3.346 & 0.744 & 8 & 6 \\
\hline Cigarette & 3.254 & 0.689 & 3.296 & 0.629 & 3.481 & 0.853 & 9 & 25 \\
\hline Score & 3.259 & 0.543 & 3.214 & 0.523 & 3.235 & 0.746 & 5 & 66 \\
\hline Job & 3.259 & 0.533 & 3.224 & 0.508 & 3.333 & 0.548 & 3 & 238 \\
\hline Fence & 3.261 & 0.650 & 3.439 & 0.690 & 3.605 & 0.817 & 5 & 30 \\
\hline Bicycle & 3.265 & 0.566 & 3.255 & 0.543 & 3.160 & 0.601 & 7 & 5 \\
\hline Stick & 3.265 & 0.632 & 3.204 & 0.536 & 3.469 & 0.654 & 5 & 39 \\
\hline Decayed & 3.271 & 0.548 & 3.173 & 0.557 & 3.296 & 0.697 & 7 & 4 \\
\hline Stapler & 3.276 & 0.508 & 3.316 & 0.619 & 3.494 & 0.673 & 7 & 1 \\
\hline Messy & 3.276 & 0.637 & 3.388 & 0.636 & 3.321 & 0.878 & 5 & 3 \\
\hline Rude & 3.282 & 0.682 & 3.316 & 0.585 & 3.358 & 0.763 & 4 & 6 \\
\hline Frog & 3.282 & 0.656 & 3.275 & 0.639 & 3.222 & 0.775 & 4 & 1 \\
\hline Horn & 3.282 & 0.602 & 3.214 & 0.613 & 3.346 & 0.727 & 4 & 31 \\
\hline Monotone & 3.288 & 0.622 & 3.122 & 0.614 & 3.358 & 0.795 & 8 & 3 \\
\hline
\end{tabular}


APPENDIX (Continued)

\begin{tabular}{|c|c|c|c|c|c|c|c|c|}
\hline \multicolumn{9}{|c|}{ Table A2 (Continued) } \\
\hline \multirow[b]{2}{*}{ Word } & \multicolumn{2}{|c|}{ NJ } & \multicolumn{2}{|c|}{$\mathrm{NC}$} & \multicolumn{2}{|c|}{$\mathrm{TX}$} & \multirow{2}{*}{$\begin{array}{l}\text { No. of } \\
\text { Letters }\end{array}$} & \multirow[b]{2}{*}{ Frequency } \\
\hline & $M$ & $S D$ & $M$ & $S D$ & $M$ & $S D$ & & \\
\hline Wagon & 3.288 & 0.604 & 3.398 & 0.587 & 3.272 & 0.707 & 5 & 55 \\
\hline Erupt & 3.288 & 0.649 & 3.265 & 0.529 & 3.469 & 0.654 & 5 & 2 \\
\hline Fish & 3.288 & 0.649 & 3.276 & 0.605 & 3.235 & 0.712 & 4 & 35 \\
\hline Roof & 3.293 & 0.606 & 3.235 & 0.571 & 3.383 & 0.663 & 4 & 59 \\
\hline Demand & 3.318 & 0.756 & 3.265 & 0.635 & 3.296 & 0.887 & 6 & 102 \\
\hline Earthquake & 3.322 & 0.651 & 3.378 & 0.711 & 3.444 & 0.707 & 10 & 9 \\
\hline Armadillo & 3.322 & 0.642 & 3.367 & 0.599 & 3.383 & 0.663 & 9 & 2 \\
\hline Loud & 3.310 & 0.730 & 3.275 & 0.513 & 3.407 & 0.848 & 4 & 20 \\
\hline Stagger & 3.310 & 0.593 & 3.245 & 0.478 & 3.370 & 0.749 & 7 & 2 \\
\hline Sock & 3.327 & 0.626 & 3.357 & 0.596 & 3.420 & 0.756 & 4 & 4 \\
\hline Lightning & 3.333 & 0.712 & 3.429 & 0.658 & 3.481 & 0.691 & 9 & 14 \\
\hline Hump & 3.355 & 0.770 & 3.224 & 0.601 & 3.346 & 0.793 & 4 & 2 \\
\hline Income & 3.355 & 0.576 & 3.275 & 0.729 & 3.518 & 0.673 & 6 & 109 \\
\hline Wheel & 3.361 & 0.578 & 3.418 & 0.641 & 3.407 & 0.587 & 5 & 56 \\
\hline Navigate & 3.367 & 0.549 & 3.408 & 0.623 & 3.506 & 0.744 & 8 & 1 \\
\hline Animal & 3.372 & 0.671 & 3.337 & 0.786 & 3.531 & 0.808 & 6 & 68 \\
\hline Forest & 3.378 & 0.681 & 3.316 & 0.754 & 3.444 & 0.894 & 6 & 66 \\
\hline Coal & 3.378 & 0.672 & 3.418 & 0.573 & 3.444 & 0.758 & 4 & 32 \\
\hline Electric & 3.384 & 0.656 & 3.429 & 0.609 & 3.531 & 0.776 & 8 & 68 \\
\hline Pension & 3.397 & 0.595 & 3.194 & 0.531 & 3.432 & 0.611 & 7 & 13 \\
\hline Mutiny & 3.401 & 0.684 & 3.429 & 0.609 & 3.457 & 0.725 & 6 & 3 \\
\hline Stone & 3.406 & 0.677 & 3.338 & 0.586 & 3.494 & 0.691 & 5 & 58 \\
\hline Cards & 3.412 & 0.669 & 3.388 & 0.741 & 3.432 & 0.688 & 5 & 36 \\
\hline Overbearing & 3.429 & 0.902 & 3.571 & 0.837 & 3.679 & 0.985 & 11 & 2 \\
\hline Bold & 3.435 & 0.751 & 3.459 & 0.676 & 3.444 & 0.866 & 4 & 21 \\
\hline Trash & 3.457 & 0.698 & 3.357 & 0.579 & 3.333 & 0.758 & 5 & 2 \\
\hline Toilet & 3.480 & 0.784 & 3.469 & 0.763 & 3.407 & 0.919 & 6 & 13 \\
\hline Eagle & 3.485 & 0.658 & 3.316 & 0.652 & 3.284 & 0.810 & 5 & 5 \\
\hline Boat & 3.491 & 0.604 & 3.449 & 0.720 & 3.580 & 0.788 & 4 & 72 \\
\hline
\end{tabular}

Table A3

Masculine Words (3.5 - 5)

\begin{tabular}{|c|c|c|c|c|c|c|c|c|}
\hline \multirow[b]{2}{*}{ Word } & \multicolumn{2}{|c|}{$\mathrm{NJ}$} & \multicolumn{2}{|c|}{$\mathrm{NC}$} & \multicolumn{2}{|c|}{ TX } & \multirow{2}{*}{$\begin{array}{l}\text { No. of } \\
\text { Letters }\end{array}$} & \multirow[b]{2}{*}{ Frequency } \\
\hline & $M$ & $S D$ & $M$ & $S D$ & $M$ & $S D$ & & \\
\hline Bark & 3.502 & 0.700 & 3.402 & 0.672 & 3.654 & 0.854 & 4 & 14 \\
\hline Rebellious & 3.508 & 0.708 & 3.724 & 0.670 & 3.704 & 0.828 & 10 & 2 \\
\hline Hell & 3.531 & 0.707 & 3.500 & 0.692 & 3.617 & 0.902 & 4 & 95 \\
\hline Chess & 3.548 & 0.714 & 3.510 & 0.646 & 3.605 & 0.753 & 5 & 3 \\
\hline Dark & 3.553 & 0.767 & 3.408 & 0.716 & 3.642 & 0.899 & 4 & 185 \\
\hline Angry & 3.559 & 0.721 & 3.500 & 0.677 & 3.778 & 0.806 & 5 & 45 \\
\hline Professor & 3.559 & 0.655 & 3.673 & 0.685 & 3.716 & 0.762 & 9 & 57 \\
\hline Helicopter & 3.570 & 0.645 & 3.546 & 0.750 & 3.728 & 0.775 & 10 & 1 \\
\hline Rocket & 3.576 & 0.750 & 3.571 & 0.673 & 3.753 & 0.859 & 6 & 7 \\
\hline Courageous & 3.593 & 0.821 & 3.541 & 0.676 & 3.543 & 0.807 & 10 & 4 \\
\hline Athletic & 3.598 & 0.785 & 3.622 & 0.650 & 3.716 & 0.794 & 8 & 18 \\
\hline Blue & 3.598 & 0.847 & 3.500 & 0.777 & 3.729 & 0.962 & 4 & 143 \\
\hline Lawyer & 3.604 & 0.683 & 3.724 & 0.700 & 3.852 & 0.808 & 6 & 43 \\
\hline Lasso & 3.604 & 0.777 & 3.639 & 0.780 & 3.691 & 0.846 & 5 & 2 \\
\hline Reckless & 3.610 & 0.594 & 3.490 & 0.646 & 3.605 & 0.736 & 8 & 9 \\
\hline Crude & 3.610 & 0.754 & 3.480 & 0.707 & 3.679 & 0.906 & 5 & 15 \\
\hline Locomotive & 3.615 & 0.690 & 3.602 & 0.728 & 3.605 & 0.847 & 10 & 2 \\
\hline Arrogant & 3.621 & 0.774 & 3.490 & 0.646 & 3.691 & 0.752 & 8 & 2 \\
\hline Gym & 3.621 & 0.774 & 3.612 & 0.636 & 3.593 & 0.787 & 3 & 2 \\
\hline Darts & 3.632 & 0.711 & 3.490 & 0.579 & 3.605 & 0.769 & 5 & 1 \\
\hline Tall & 3.632 & 0.735 & 3.827 & 0.689 & 3.778 & 0.806 & 4 & 55 \\
\hline Domineering & 3.632 & 0.856 & 3.546 & 0.736 & 3.778 & 0.922 & 11 & 2 \\
\hline Hostile & 3.632 & 0.772 & 3.694 & 0.709 & 3.876 & 0.797 & 7 & 19 \\
\hline Tattoo & 3.638 & 0.710 & 3.704 & 0.706 & 3.741 & 0.803 & 6 & 1 \\
\hline Forceful & 3.655 & 0.715 & 3.663 & 0.591 & 3.839 & 0.715 & 8 & 8 \\
\hline Snake & 3.655 & 0.797 & 3.449 & 0.690 & 3.580 & 0.934 & 5 & 44 \\
\hline Judge & 3.661 & 0.681 & 3.684 & 0.667 & 3.827 & 0.755 & 5 & 77 \\
\hline Billiards & 3.687 & 0.732 & 3.704 & 0.629 & 3.753 & 0.767 & 9 & 1 \\
\hline Tornado & 3.689 & 0.730 & 3.612 & 0.808 & 3.617 & 0.767 & 7 & 1 \\
\hline Violent & 3.700 & 0.678 & 3.612 & 0.683 & 3.728 & 0.837 & 7 & 33 \\
\hline
\end{tabular}


APPENDIX (Continued)

\begin{tabular}{|c|c|c|c|c|c|c|c|c|}
\hline \multicolumn{9}{|c|}{ Table A3 (Continued) } \\
\hline \multirow[b]{2}{*}{ Word } & \multicolumn{2}{|c|}{ NJ } & \multicolumn{2}{|c|}{$\mathrm{NC}$} & \multicolumn{2}{|c|}{$\mathrm{TX}$} & \multirow{2}{*}{$\begin{array}{l}\text { No. of } \\
\text { Letters }\end{array}$} & \multirow[b]{2}{*}{ Frequency } \\
\hline & $M$ & $S D$ & $M$ & $S D$ & $M$ & $S D$ & & \\
\hline Powerful & 3.706 & 0.807 & 3.806 & 0.727 & 3.753 & 0.902 & 8 & 63 \\
\hline Jeep & 3.711 & 0.732 & 3.806 & 0.698 & 3.753 & 0.859 & 4 & 16 \\
\hline Competitive & 3.723 & 0.773 & 3.775 & 0.696 & 3.852 & 0.853 & 11 & 31 \\
\hline Garage & 3.728 & 0.749 & 3.633 & 0.664 & 3.839 & 0.843 & 6 & 21 \\
\hline Strapping & 3.733 & 0.836 & 3.531 & 0.789 & 3.642 & 0.826 & 9 & 3 \\
\hline Vulgar & 3.734 & 0.864 & 3.796 & 0.641 & 3.790 & 0.720 & 6 & 7 \\
\hline Destructive & 3.734 & 0.732 & 3.701 & 0.615 & 3.876 & 0.871 & 11 & 25 \\
\hline Briefcase & 3.740 & 0.639 & 3.684 & 0.619 & 3.938 & 0.747 & 9 & 1 \\
\hline Bridegroom & 3.744 & 1.268 & 3.622 & 1.206 & 3.568 & 1.369 & 10 & 3 \\
\hline Rough & 3.745 & 0.713 & 3.765 & 0.700 & 3.852 & 0.776 & 5 & 41 \\
\hline Surgeon & 3.745 & 0.721 & 3.775 & 0.696 & 3.741 & 0.771 & 7 & 11 \\
\hline Doctor & 3.768 & 0.688 & 3.837 & 0.713 & 3.790 & 0.847 & 6 & 100 \\
\hline Wrench & 3.779 & 0.840 & 3.663 & 0.884 & 3.914 & 0.869 & 6 & 1 \\
\hline Pliers & 3.796 & 0.785 & 3.633 & 0.709 & 3.716 & 0.778 & 6 & 1 \\
\hline Tough & 3.807 & 0.696 & 3.775 & 0.725 & 3.889 & 0.851 & 5 & 36 \\
\hline Wallet & 3.807 & 0.744 & 3.939 & 0.757 & 4.000 & 0.866 & 6 & 6 \\
\hline Sports & 3.819 & 0.762 & 3.816 & 0.663 & 3.938 & 0.812 & 6 & 49 \\
\hline Strong & 3.830 & 0.702 & 3.939 & 0.701 & 3.901 & 0.831 & 6 & 202 \\
\hline Drill & 3.841 & 0.781 & 3.796 & 0.773 & 3.876 & 0.857 & 5 & 33 \\
\hline Screwdriver & 3.847 & 0.710 & 3.796 & 0.673 & 4.000 & 0.742 & 11 & 1 \\
\hline Astronaut & 3.892 & 0.686 & 3.786 & 0.662 & 3.827 & 0.738 & 9 & 2 \\
\hline Hero & 3.898 & 0.826 & 3.755 & 0.897 & 3.753 & 0.942 & 4 & 52 \\
\hline Skateboard & 3.904 & 0.654 & 3.888 & 0.607 & 4.000 & 0.775 & 10 & 1 \\
\hline Pistol & 3.915 & 0.721 & 3.939 & 0.671 & 3.963 & 0.766 & 6 & 27 \\
\hline Pilot & 3.915 & 0.681 & 3.908 & 0.761 & 4.037 & 0.858 & 5 & 44 \\
\hline Fight & 3.932 & 0.815 & 3.969 & 0.818 & 3.963 & 0.858 & 5 & 98 \\
\hline God & 3.932 & 0.920 & 3.969 & 0.913 & 3.975 & 1.012 & 3 & 318 \\
\hline Truck & 3.932 & 0.719 & 3.867 & 0.683 & 3.975 & 0.724 & 5 & 57 \\
\hline Dentist & 3.937 & 0.754 & 3.969 & 0.710 & 4.000 & 0.775 & 7 & 12 \\
\hline Police & 3.937 & 0.604 & 3.949 & 0.598 & 3.975 & 0.689 & 6 & 155 \\
\hline Dominant & 3.943 & 0.720 & 3.939 & 0.715 & 4.000 & 0.894 & 8 & 65 \\
\hline Exterminator & 3.949 & 0.763 & 3.847 & 0.709 & 4.148 & 0.882 & 12 & 1 \\
\hline Tractor & 3.966 & 0.797 & 4.051 & 0.751 & 4.148 & 0.776 & 7 & 24 \\
\hline Engineer & 3.966 & 0.782 & 3.867 & 0.713 & 3.988 & 0.782 & 8 & 42 \\
\hline Bicep & 4.005 & 0.726 & 3.990 & 0.634 & 4.000 & 0.689 & 5 & 1 \\
\hline Devil & 4.016 & 0.828 & 3.898 & 0.806 & 4.123 & 0.871 & 5 & 25 \\
\hline Aggressive & 4.022 & 0.573 & 4.051 & 0.632 & 4.185 & 0.654 & 10 & 17 \\
\hline Chiseled & 4.022 & 0.706 & 3.786 & 0.721 & 3.864 & 0.833 & 8 & 2 \\
\hline Politician & 4.028 & 0.741 & 3.949 & 0.694 & 3.963 & 0.766 & 10 & 13 \\
\hline Poker & 4.028 & 0.686 & 4.051 & 0.709 & 4.160 & 0.697 & 5 & 6 \\
\hline Master & 4.039 & 0.725 & 4.051 & 0.648 & 4.074 & 0.932 & 6 & 72 \\
\hline Fishing & 4.050 & 0.676 & 4.133 & 0.636 & 4.049 & 0.757 & 7 & 32 \\
\hline Hammer & 4.050 & 0.770 & 3.918 & 0.795 & 4.012 & 0.901 & 6 & 9 \\
\hline Burly & 4.067 & 0.822 & 3.794 & 0.853 & 4.210 & 0.862 & 5 & 3 \\
\hline Prisoner & 4.090 & 0.668 & 3.990 & 0.666 & 4.185 & 0.709 & 8 & 7 \\
\hline Brawny & 4.096 & 0.751 & 3.796 & 0.837 & 4.099 & 0.982 & 6 & 1 \\
\hline Veteran & 4.146 & 0.731 & 4.082 & 0.713 & 4.037 & 0.828 & 7 & 27 \\
\hline Beer & 4.146 & 0.691 & 4.194 & 0.727 & 4.259 & 0.721 & 4 & 34 \\
\hline Hairy & 4.152 & 0.771 & 4.000 & 0.718 & 4.049 & 0.723 & 5 & 5 \\
\hline War & 4.169 & 0.734 & 4.071 & 0.749 & 4.272 & 0.806 & 3 & 464 \\
\hline Hunting & 4.169 & 0.726 & 4.020 & 0.703 & 4.272 & 0.758 & 7 & 34 \\
\hline Trousers & 4.186 & 0.686 & 4.031 & 0.766 & 4.086 & 0.745 & 8 & 7 \\
\hline Janitor & 4.186 & 0.669 & 3.929 & 0.630 & 3.975 & 0.724 & 7 & 4 \\
\hline Carpenter & 4.203 & 0.717 & 4.071 & 0.707 & 4.025 & 0.894 & 9 & 6 \\
\hline Umpire & 4.237 & 0.746 & 4.102 & 0.739 & 4.173 & 0.818 & 6 & 1 \\
\hline Hockey & 4.237 & 0.811 & 4.194 & 0.683 & 4.197 & 0.765 & 6 & 1 \\
\hline Army & 4.248 & 0.765 & 4.163 & 0.621 & 4.160 & 0.813 & 4 & 132 \\
\hline Cigars & 4.276 & 0.696 & 4.173 & 0.746 & 4.272 & 0.725 & 6 & 2 \\
\hline President & 4.288 & 0.683 & 4.398 & 0.638 & 4.296 & 0.715 & 9 & 382 \\
\hline Sheriff & 4.288 & 0.640 & 4.224 & 0.634 & 4.296 & 0.732 & 7 & 20 \\
\hline Mechanic & 4.322 & 0.756 & 4.204 & 0.688 & 4.296 & 0.732 & 8 & 5 \\
\hline Muscular & 4.333 & 0.704 & 4.286 & 0.673 & 4.272 & 0.791 & 8 & 16 \\
\hline Bald & 4.350 & 0.649 & 4.153 & 0.778 & 4.321 & 0.755 & 4 & 5 \\
\hline Baseball & 4.355 & 0.709 & 4.306 & 0.738 & 4.259 & 0.771 & 8 & 57 \\
\hline Plumber & 4.361 & 0.677 & 4.286 & 0.689 & 4.296 & 0.828 & 7 & 4 \\
\hline
\end{tabular}


APPENDIX (Continued)

Table A3 (Continued)

\begin{tabular}{|c|c|c|c|c|c|c|c|c|}
\hline \multirow[b]{2}{*}{ Word } & \multicolumn{2}{|c|}{ NJ } & \multicolumn{2}{|c|}{$\mathrm{NC}$} & \multicolumn{2}{|c|}{ TX } & \multirow{2}{*}{$\begin{array}{l}\text { No. of } \\
\text { Letters }\end{array}$} & \multirow[b]{2}{*}{ Frequency } \\
\hline & $M$ & $S D$ & $M$ & $S D$ & $M$ & $S D$ & & \\
\hline Gigalo & 4.384 & 0.818 & 4.073 & 0.861 & 4.444 & 0.806 & 6 & 1 \\
\hline Bowtie & 4.389 & 0.738 & 4.337 & 0.673 & 4.321 & 0.849 & 6 & 1 \\
\hline Football & 4.429 & 0.712 & 4.459 & 0.629 & 4.605 & 0.540 & 8 & 36 \\
\hline Fraternity & 4.717 & 0.706 & 4.653 & 0.719 & 4.259 & 1.253 & 10 & 6 \\
\hline Soldier & 4.429 & 0.645 & 4.337 & 0.657 & 4.321 & 0.772 & 7 & 39 \\
\hline Boxing & 4.446 & 0.689 & 4.347 & 0.690 & 4.395 & 0.736 & 6 & 1 \\
\hline Necktie & 4.463 & 0.699 & 4.347 & 0.761 & 4.247 & 0.902 & 7 & 2 \\
\hline Duke & 4.497 & 0.700 & 4.082 & 0.991 & 4.284 & 0.965 & 4 & 11 \\
\hline Sideburns & 4.502 & 0.640 & 4.449 & 0.660 & 4.432 & 0.670 & 9 & 1 \\
\hline Cologne & 4.525 & 0.666 & 4.418 & 0.772 & 4.136 & 1.081 & 7 & 9 \\
\hline Cowboy & 4.593 & 0.596 & 4.551 & 0.720 & 4.531 & 0.743 & 6 & 16 \\
\hline Pirate & 4.593 & 0.615 & 4.398 & 0.714 & 4.395 & 0.817 & 6 & 4 \\
\hline Goatee & 4.661 & 0.638 & 4.490 & 0.707 & 4.531 & 0.867 & 6 & 1 \\
\hline Wrestling & 4.672 & 0.548 & 4.684 & 0.619 & 4.741 & 0.468 & 9 & 1 \\
\hline Priest & 4.683 & 0.613 & 4.459 & 0.629 & 4.481 & 0.691 & 6 & 16 \\
\hline Beard & 4.717 & 0.553 & 4.571 & 0.592 & 4.642 & 0.730 & 5 & 26 \\
\hline Handsome & 4.717 & 0.521 & 4.643 & 0.579 & 4.593 & 0.587 & 8 & 40 \\
\hline Mustache & 4.785 & 0.463 & 4.653 & 0.520 & 4.691 & 0.625 & 8 & 5 \\
\hline Prince & 4.819 & 0.523 & 4.643 & 0.707 & 4.453 & 0.949 & 6 & 33 \\
\hline Bachelor & 4.819 & 0.544 & 4.786 & 0.460 & 4.728 & 0.742 & 8 & 6 \\
\hline King & 4.847 & 0.616 & 4.806 & 0.653 & 4.790 & 0.646 & 4 & 88 \\
\hline Testosterone & 4.881 & 0.429 & 4.826 & 0.455 & 4.753 & 0.582 & 12 & 1 \\
\hline Husband & 4.881 & 0.535 & 4.939 & 0.281 & 4.864 & 0.379 & 7 & 131 \\
\hline Father & 4.904 & 0.435 & 4.888 & 0.451 & 4.889 & 0.353 & 6 & 183 \\
\hline Brother & 4.904 & 0.422 & 4.765 & 0.729 & 4.790 & 0.627 & 7 & 73 \\
\hline Uncle & 4.943 & 0.254 & 4.878 & 0.482 & 4.926 & 0.346 & 5 & 5 \\
\hline
\end{tabular}

Table A4

Mean Ratings by Sex of Participant for Items That Differed Reliably in Their Gender Categorization for the New Jersey Sample

\begin{tabular}{|c|c|c|c|c|c|c|c|c|c|c|c|}
\hline \multirow[b]{2}{*}{ Item } & \multicolumn{2}{|c|}{$\begin{array}{l}\text { Group } \\
\text { Rating }\end{array}$} & \multicolumn{3}{|c|}{$\begin{array}{c}\text { Male Participants' } \\
\text { Rating }\end{array}$} & \multicolumn{3}{|c|}{$\begin{array}{c}\text { Female Participants' } \\
\text { Rating } \\
\end{array}$} & \multirow[b]{2}{*}{$t$} & \multirow[b]{2}{*}{$d f$} & \multirow[b]{2}{*}{$p$} \\
\hline & $M$ & $S D$ & $M$ & $S D$ & & $M$ & $S D$ & & & & \\
\hline \multicolumn{12}{|c|}{ Feminine Words } \\
\hline Desire & 2.435 & 0.728 & 2.607 & 0.802 & $\mathrm{~N}$ & 2.355 & 0.681 & $\mathrm{~F}$ & 2.034 & 93.146 & .045 \\
\hline Patient & 2.457 & 0.730 & 2.661 & 0.695 & $\mathrm{~N}$ & 2.364 & 0.730 & $\mathrm{~F}$ & 2.603 & 112.176 & .011 \\
\hline Star & 2.457 & 0.722 & 2.679 & 0.543 & $\mathrm{~N}$ & 2.355 & 0.773 & $\mathrm{~F}$ & 3.200 & 147.224 & .002 \\
\hline Champagne & 2.446 & 0.745 & 2.679 & 0.690 & $\mathrm{~N}$ & 2.339 & 0.748 & $\mathrm{~F}$ & 2.965 & 115.359 & .004 \\
\hline Heaven & 2.440 & 0.721 & 2.696 & 0.569 & $\mathrm{~N}$ & 2.322 & 0.755 & $\mathrm{~F}$ & 3.650 & 138.800 & $<.001$ \\
\hline \multicolumn{12}{|c|}{ Neutral Words } \\
\hline Beach & 2.565 & 0.619 & 2.786 & 0.530 & $\mathrm{~N}$ & 2.463 & 0.633 & $\mathrm{~F}$ & 3.540 & 126.430 & .001 \\
\hline Color & 2.598 & 0.623 & 2.821 & 0.508 & $\mathrm{~N}$ & 2.496 & 0.647 & $\mathrm{~F}$ & 3.623 & 133.901 & $<.001$ \\
\hline Boat & 3.491 & 0.604 & 3.304 & 0.537 & $\mathrm{~N}$ & 3.578 & 0.616 & M & 3.022 & 121.738 & .003 \\
\hline Faithful & 2.508 & 0.666 & 2.679 & 0.543 & $\mathrm{~N}$ & 2.430 & 0.705 & $\mathrm{~F}$ & 2.570 & 136.295 & .011 \\
\hline Pleasing & 2.502 & 0.716 & 2.679 & 0.606 & $\mathrm{~N}$ & 2.421 & 0.750 & $\mathrm{~F}$ & 2.428 & 130.504 & .017 \\
\hline \multicolumn{12}{|c|}{ Masculine Words } \\
\hline Darts & 3.632 & 0.711 & 3.464 & 0.713 & $\mathrm{~N}$ & 3.711 & 0.700 & M & 2.151 & 105.516 & .034 \\
\hline Snake & 3.655 & 0.797 & 3.464 & 0.785 & $\mathrm{~N}$ & 3.744 & 0.791 & M & 2.197 & 107.845 & .030 \\
\hline Dark & 3.553 & 0.767 & 3.339 & 0.611 & $\mathrm{~N}$ & 3.653 & 0.813 & M & 2.846 & 139.239 & .005 \\
\hline Domineering & 3.632 & 0.856 & 3.429 & 0.735 & $\mathrm{~N}$ & 3.727 & 0.894 & $\mathrm{M}$ & 2.343 & 128.545 & .021 \\
\hline Rocket & 3.576 & 0.750 & 3.411 & 0.708 & $\mathrm{~N}$ & 3.653 & 0.761 & M & 2.067 & 114.508 & .041 \\
\hline Arrogant & 3.621 & 0.774 & 3.357 & 0.672 & $\mathrm{~N}$ & 3.744 & 0.791 & $\mathrm{M}$ & 3.360 & 124.593 & .001 \\
\hline Crude & 3.610 & 0.754 & 3.375 & 0.702 & $\mathrm{~N}$ & 3.719 & 0.755 & $\mathrm{M}$ & 2.958 & 114.589 & .004 \\
\hline Bridegroom & 3.744 & 1.268 & 3.291 & 1.286 & $\mathrm{~N}$ & 3.950 & 1.210 & $\mathrm{M}$ & 3.211 & 98.974 & .002 \\
\hline Strapping & 3.733 & 0.836 & 3.464 & 0.852 & $\mathrm{~N}$ & 3.858 & 0.802 & M & 2.911 & 101.866 & .004 \\
\hline
\end{tabular}

Note-The category that corresponds to the mean rating is presented along with the mean rating. M, male category; F, female category; $\mathrm{N}$, neutral category. 
APPENDIX (Continued)

\begin{tabular}{|c|c|c|c|c|c|c|c|c|c|c|c|}
\hline \multicolumn{12}{|c|}{$\begin{array}{c}\text { Table A5 } \\
\text { Mean Ratings by Sex of Participant for Items That Differed Reliably in } \\
\text { Their Gender Categorization for the North Carolina Sample }\end{array}$} \\
\hline \multirow[b]{2}{*}{ Item } & \multicolumn{2}{|c|}{$\begin{array}{l}\text { Group } \\
\text { Rating } \\
\end{array}$} & \multicolumn{3}{|c|}{$\begin{array}{l}\text { Male Participants' } \\
\text { Rating }\end{array}$} & \multicolumn{3}{|c|}{$\begin{array}{l}\text { Female Participants' } \\
\text { Rating }\end{array}$} & \multirow[b]{2}{*}{$t$} & \multirow[b]{2}{*}{$d f$} & \multirow[b]{2}{*}{$p$} \\
\hline & $M$ & $S D$ & $M$ & $S D$ & & $M$ & $S D$ & & & & \\
\hline \multicolumn{12}{|c|}{ Feminine Words } \\
\hline Springtime & 2.541 & 0.595 & 2.680 & 0.513 & $\mathrm{~N}$ & 2.396 & 0.644 & $\mathrm{~F}$ & 2.411 & 89.745 & .018 \\
\hline \multicolumn{12}{|c|}{ Neutral Words } \\
\hline Toilet & 3.469 & 0.763 & 3.300 & 0.839 & $\mathrm{~N}$ & 3.646 & 0.635 & M & 2.306 & 91.128 & .023 \\
\hline Bold & 3.459 & 0.676 & 3.600 & 0.782 & M & 3.312 & 0.512 & $\mathrm{~N}$ & 2.161 & 84.842 & .034 \\
\hline Stove & 2.571 & 0.673 & 2.440 & 0.704 & $\mathrm{~F}$ & 2.708 & 0.617 & $\mathrm{~N}$ & 2.007 & 95.224 & .048 \\
\hline \multicolumn{12}{|c|}{ Masculine Words } \\
\hline Courageous & 3.541 & 0.676 & 3.680 & 0.653 & M & 3.396 & 0.676 & $\mathrm{~N}$ & 2.116 & 95.446 & .037 \\
\hline Gym & 3.612 & 0.636 & 3.740 & 0.664 & M & 3.479 & 0.583 & $\mathrm{~N}$ & 2.068 & 95.251 & .041 \\
\hline Bridegroom & 3.622 & 1.206 & 3.380 & 1.338 & $\mathrm{~N}$ & 3.875 & 1.003 & M & 2.077 & 90.714 & .041 \\
\hline
\end{tabular}

Table A6

Mean Ratings by Sex of Participant for Items That Differed Reliably in Their Gender Categorization for the Texas Sample

\begin{tabular}{|c|c|c|c|c|c|c|c|c|c|c|c|}
\hline \multirow[b]{2}{*}{ Item } & \multicolumn{2}{|c|}{$\begin{array}{l}\text { Group } \\
\text { Rating }\end{array}$} & \multicolumn{3}{|c|}{$\begin{array}{c}\text { Male Participants' } \\
\text { Rating }\end{array}$} & \multicolumn{3}{|c|}{$\begin{array}{c}\text { Female Participants' } \\
\text { Rating }\end{array}$} & \multirow[b]{2}{*}{$t$} & \multirow[b]{2}{*}{$d f$} & \multirow[b]{2}{*}{$p$} \\
\hline & $M$ & $S D$ & $M$ & $S D$ & & $M$ & $S D$ & & & & \\
\hline \multicolumn{12}{|c|}{ Feminine Words } \\
\hline Patient & 2.346 & 0.824 & 2.667 & 0.917 & $\mathrm{~N}$ & 2.210 & 0.750 & $\mathrm{~F}$ & 2.153 & 36.582 & .038 \\
\hline Laundry & 2.383 & 0.874 & 2.708 & 0.955 & $\mathrm{~N}$ & 2.246 & 0.808 & $\mathrm{~F}$ & 2.081 & 37.566 & .044 \\
\hline Star & 2.346 & 0.824 & 2.667 & 0.917 & $\mathrm{~N}$ & 2.210 & 0.750 & $\mathrm{~F}$ & 2.153 & 36.582 & .038 \\
\hline \multicolumn{12}{|c|}{ Neutral Words } \\
\hline Gallery & 2.593 & 0.755 & 2.917 & 0.881 & $\mathrm{~N}$ & 2.456 & 0.657 & $\mathrm{~F}$ & 2.306 & 34.258 & .027 \\
\hline Stone & 3.494 & 0.691 & 3.792 & 0.884 & M & 3.368 & .555 & $\mathrm{~N}$ & 2.173 & 30.931 & .038 \\
\hline Thanks & 2.580 & 0.589 & 2.792 & 0.509 & $\mathrm{~N}$ & 2.491 & 0.601 & $\mathrm{~F}$ & 2.295 & 50.771 & .026 \\
\hline Conniving & 2.802 & 1.005 & 2.375 & 0.970 & $\mathrm{~F}$ & 2.982 & 0.973 & $\mathrm{~N}$ & 2.572 & 43.418 & .014 \\
\hline \multicolumn{12}{|c|}{ Masculine Words } \\
\hline Reckless & 3.605 & 0.736 & 3.208 & 0.833 & $\mathrm{~N}$ & 3.772 & 0.627 & M & 2.978 & 34.488 & .005 \\
\hline Bridegroom & 3.568 & 1.369 & 2.958 & 1.429 & $\mathrm{~N}$ & 3.825 & 1.269 & M & 2.573 & 39.057 & .014 \\
\hline
\end{tabular}

(Manuscript received October 22, 2003; revision accepted for publication June 17, 2004.) 